\title{
Conventional versus ultrasound and microwave assisted synthesis: Some new environmentally friendly functionalized picolinium-based ionic liquids with potential antibacterial activity
}

MOUSLIM MESSALI

Chemistry Department

Faculty of Science, Taibah University

30002, Al-Madina Al-Munawara

Saudi Arabia

\begin{abstract}
A green chemistry approach has been adopted for the synthesis of thirty-four new picolinium-based ionic liquids using microwave (MW) and ultrasound (US) irradiation as well as conventional thermal heating. Their structures were confirmed by FT-IR, ${ }^{1} \mathrm{H}$ NMR, ${ }^{13} \mathrm{C}$ NMR, ${ }^{11} \mathrm{~B}$ NMR, ${ }^{19} \mathrm{~F}$ NMR, ${ }^{31} \mathrm{P}$ NMR, mass spectra and elemental analyses. The antimicrobial profile of the novel ionic liquids was evaluated and the minimum inhibitory concentration $(M I C)$ showed their moderate to low antimicrobial activity against eight types of human pathogens.
\end{abstract}

Keywords: picolinium-based ionic liquids, ultrasound irradiation, microwave irradiation, green procedure, antibacterial activity

In the past years, ionic liquids (ILs) created a new set of challenges to chemists and chemical engineers and gained considerable interest as excellent alternatives to volatile organic compounds (VOCs) due to their unique properties such as negligible vapor pressure, excellent thermal and chemical stability in a wide temperature range, no flammability, high ionic conductivity and solvation ability (1).

ILs are applicable in various fields, e.g., as solvents in organic synthesis (2), as media for electrodeposition of metals $(3,4)$, as corrosion inhibitors (5-9), as electrolytes for electrochemical devices such as batteries $(10,11)$, as catalysts (12), in fuel cells (13), in polymer science (14), in dye-sensitized solar cells (15).

Separately, ultrasound (US) and microwave (MW) irradiation have gained popularity as powerful tools in organic chemistry (16-20), offering some important advantages such as significantly faster reaction times, increase in yield and selectivity, lower costs and simplicity in handling and processing.

Contrary to conventional heating, in which energy must be first conducted through the walls of the vessel containing the reactants, microwave irradiation heats the contents

\footnotetext{
*Correspondence: e-mail: mouslim@mail.be
} 
directly, allowing the temperature to raise much faster, thus boosting reaction rates. Another characteristic of microwaves is their ability to superheat solvents to a temperature well in excess of their normal boiling points; this is due to the even spread of heat through the liquid, allowing it to reach higher temperature before bubbles form (21).

As continuation of our interest in the development of green chemical processes (2224), the synthesis of a variety of new picolinium-based ionic liquids under ultrasound and microwave irradiation was investigated carefully, and the protocol was then compared with similar preparation using conventional heating.

To the best of our knowledge, only 1-(5-carboxypentyl)-4-methylpyridinium bromide (5) was previously prepared by conventional methods (25); its preparation under ultrasound (US) or microwave irradiation (MW) has never been disclosed.

Further, in continuation of our recent work showing that some ILs possess promising biological activities $(26,27)$, the in vitro antibacterial activities of some newly obtained ILs were tested.

\section{EXPERIMENTAL}

\section{Apparatus}

All new compounds were synthesized and characterized by ${ }^{1} \mathrm{H}$ NMR, ${ }^{13} \mathrm{C}$ NMR, ${ }^{19} \mathrm{~F}$ NMR, ${ }^{11} \mathrm{~B}$ NMR, ${ }^{31} \mathrm{P}$ NMR, infrared spectroscopy (IR) and liquid chromatographymass spectrometry (LC-MS). ${ }^{1} \mathrm{H}$ NMR $(400 \mathrm{MHz}),{ }^{13} \mathrm{C}$ NMR $(100 \mathrm{MHz}),{ }^{19} \mathrm{~F}$ NMR $(376.5 \mathrm{MHz})$, ${ }^{31} \mathrm{P}$ NMR $(162 \mathrm{MHz})$ and ${ }^{11} \mathrm{~B}$ NMR $(128 \mathrm{MHz})$ spectra were measured in DMSO, $\mathrm{CDCl}_{3}$ or $\mathrm{D}_{2} \mathrm{O}$ at room temperature. Chemical shifts $(\delta)$ were reported in ppm relative to tetramethylsilane (TMS), which was used as an internal standard (Bruker, Switzerland). The LC-MS spectra were measured with a Micromass, LCT mass spectrometer (Agilent Technologies, Germany). IR spectra $\left(v_{\max }\right.$ in $\left.\mathrm{cm}^{-1}\right)$ were recorded in $\mathrm{NaCl}$ or $\mathrm{KBr}$ disc in a Shimadzu 8201 PC, FTIR spectrophotometer (Shimadzu, Japan). Elemental analyses were given using the 2400 Series II CHNS/O Elemental Analyzer (Perkin Elmer, USA). The ultrasound-assisted reactions were performed using a high intensity ultrasonic processor SUB Aqua 5 PlusGrant with a temperature controller $(750 \mathrm{~W})$, microprocessor controlled-2004; the ultrasonic frequency of the cleaning bath used equaled $25 \mathrm{kHz}$ (Grant Scientific, UK). The microwave-assisted reactions were performed using a controllable single-mode microwave reactor, CEM Discovery, designed for synthetic use. The reactor was equipped with a magnetic stirrer as well as pressure, temperature and power controls. The maximum operating pressure of the reactor was $2 \mathrm{MPa}$. Power and temperature ranges were 15-300 W and $60-250{ }^{\circ} \mathrm{C}$, respectively (GEM Corp., USA).

\section{General synthetic procedures}

Picolinium halides 1-5 using the conventional method (CP). - To a solution of picoline derivative ( $2 \mathrm{~g}, 0.021 \mathrm{~mol})$ in $20 \mathrm{~mL}$ toluene, alkyl halide $(0.023 \mathrm{~mol})$ was added at room temperature, followed by stirring at $80^{\circ} \mathrm{C}$ for $18 \mathrm{~h}$. Completion of the reaction was followed by separation of oil or solid from the initially obtained clear and homogenous mixture of picoline derivative and alkyl halide in toluene. The product was isolated by extraction or 
filtration to remove the unreacted starting materials and solvent. Subsequently, quaternary salt was washed with $30 \mathrm{~mL}$ of ethyl acetate. In each case, the IL/salt was finally dried at a reduced pressure to get rid of all the volatile organic compounds (see Table I).

Picolinium halides 1-5 using ultrasonic irradiation (US). - A solution of picoline derivative ( $2 \mathrm{~g}, 0.021 \mathrm{~mol})$ and the appropriate alkyl bromide $(0.023 \mathrm{~mol})$ in $10 \mathrm{~mL}$ of toluene were placed in a closed vessel and exposed to irradiation for 5 hours at $80^{\circ} \mathrm{C}$ using a sonication bath. The product was then collected as described under the conventional procedure outlined earlier.

Picolinium halides 1-5 using microwave irradiation (MW). - A solution of picoline derivative $(2 \mathrm{~g}, 0.021 \mathrm{~mol})$ and the appropriate alkyl halide $(0.023 \mathrm{~mol})$ in $10 \mathrm{~mL}$ were placed in a microwave reactor vessel and irradiated for 15 minutes at $80{ }^{\circ} \mathrm{C}$. The product was then collected as described under the conventional procedure outlined above.

Metathesis reaction of picolinium halides 1-5 leading to compounds 6-35 using the conventional method. - Quaternary salt $(0.015 \mathrm{~mol})$ was dissolved in $20 \mathrm{~mL}$ of acetonitrile to obtain a clear solution. A metal salt was added to this solution, either sodium tetrafluoroborate $\left(\mathrm{NaBF}_{4}\right)$, potassium hexafluorophosphate $\left(\mathrm{KPF}_{6}\right)$, trifluoroacetic acid sodium salt $\left(\mathrm{CF}_{3} \mathrm{COO}\right.$ $\mathrm{Na})$, sodium dicyanamide $\left[\mathrm{NaN}(\mathrm{CN})_{2}\right]$, sodium thiocyanate $(\mathrm{NaNCS})$ or sodium nitrate $\left(\mathrm{NaNO}_{3}\right)(0.018 \mathrm{~mol})$, followed by stirring at $70{ }^{\circ} \mathrm{C}$ for $3 \mathrm{~h}$. The cooled reaction mixture was filtered through Celite to remove solid metal halide, and acetonitrile was evaporated to afford the desired ionic liquid quantitatively (see Table II).

Ultrasound-assisted metathesis reaction of picolinium halides 1-5 leading to compounds 6-35. - A solution of picolinium halides $(0.015 \mathrm{~mol})$ and $\mathrm{NaBF}_{4}, \mathrm{KPF}_{6}, \mathrm{CF}_{3} \mathrm{COONa}, \mathrm{NaN}(\mathrm{CN})_{2}$, NaNCS or $\mathrm{NaNO}_{3}(0.015 \mathrm{~mol})$ in $10 \mathrm{~mL}$ of acetonitrile was placed in a closed vessel and exposed to irradiation for $45 \mathrm{~min}$ at $70{ }^{\circ} \mathrm{C}$ using a sonication bath. The product was then collected as described in the conventional procedure outlined earlier.

Microwave-assisted metathesis reaction of picolinium halides 1-5 leading to compounds 6-35. - Picolinium-halide salt $(0.015 \mathrm{~mol})$ was placed in a microwave reactor vessel, followed by the addition of $\mathrm{NaBF}_{4}, \mathrm{KPF}_{6}, \mathrm{CF}_{3} \mathrm{COONa}, \mathrm{NaN}(\mathrm{CN})_{2}, \mathrm{NaNCS}$ or $\mathrm{NaNO}_{3}(0.015 \mathrm{~mol})$ in $10 \mathrm{~mL}$ of acetonitrile. The reaction was carried out for $7 \mathrm{~min}$ at $70{ }^{\circ} \mathrm{C}$. The product was then collected as described in the conventional procedure outlined earlier.

\section{Antimicrobial activity}

Minimum inhibitory concentrations. - The antibacterial activity was measured by determination of minimum inhibitory concentration (MIC) values in a range from 0 to $1 \mathrm{mmol}$ $\mathrm{L}^{-1}$ and compared with those of some potent antibacterial compounds such as mezlocillin, amikacin and tetracycline.

Microorganisms used were four Gram-negative bacteria including Escherichia coli, Klebsiella pneumoniae, Pseudomonas aeruginosa and Acinetobacter baumannii as well as four Gram-positive bacteria including Staphylococcus aureus, Streptococcus pneumoniae, Bacillus subtilis and Bacillus cereus. These clinical isolates of microorganisms were selected because of their pathogenic properties. 
MIC was determined using the broth microdilution method based on the recommended protocol employed by the Clinical and Laboratory Standards Institute (28). Tested compounds were dissolved in sterile, distilled water and diluted to a final concentration of $2 \mathrm{mmol} \mathrm{L}^{-1}$ in Müller-Hinton broth (Becton Dickinson, USA) (29). Two-fold serially diluted test compounds were dispensed into each of the 96 wells of standard microdilution plates. The direct colony suspension method was used for inoculum preparation. Bacterial suspension was prepared by direct transfer of colonies from 24-h agar plates to MüllerHinton broth. Bacterial suspensions were adjusted using a bacterial counting chamber to contain approximately $1 \times 10^{8} \mathrm{CFU} \mathrm{mL}^{-1}$. A $50-\mu \mathrm{L}$ volume of each bacterial suspension was mixed with $50 \mu \mathrm{L}$ of a serially diluted test compound in a 96-microdilution plate according to the microdilution method (30); uninoculated wells were used as control samples. Plates were incubated at $35^{\circ} \mathrm{C}$ for $24 \mathrm{~h}$. Confirmation of $M I C$ was achieved by transfering aliquots from wells containing no growth onto nutrient agar plates and testing for colony formation upon subculturing. The obtained MIC values are means of three independent experiments and are presented in Table IV.

\section{RESULTS AND DISCUSSION}

\section{Chemistry}

The synthetic route used in this study is shown in Scheme 1. First, nucleophilic alkylation of picoline derivatives with different alkyl halides (1:1.1) was carried out using the conventional procedure (toluene, $80^{\circ} \mathrm{C}, 18 \mathrm{~h}$ ). 3-Picoline or 4-picoline played the role of nucleophile in these $\mathrm{SN}_{2}$ quaternization reactions. Picolinium halides $\mathbf{1 - 5}$ were obtained in $72-82 \%$ yield. Compared to the conventional method, ultrasonic irradiation shortened the reaction time $(5 \mathrm{~h}$ ) and gave better yield (79-84 \%) of the product (Table I). These results could be explained by the high pressure generated by the bubble collapse that prompted the reaction, as well as by the good interaction between molecules during the reaction due to vigorous agitation.

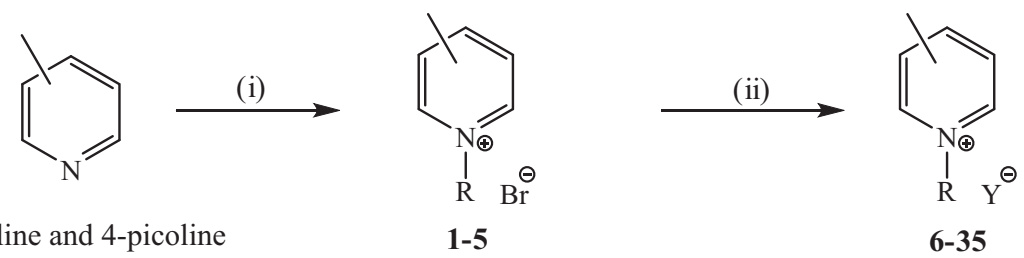

3-picoline and 4-picoline

$$
\begin{aligned}
& \mathrm{R}=\left(\mathrm{CH}_{2}\right)_{4} \mathrm{OPh},\left(\mathrm{CH}_{2}\right)_{5} \mathrm{CO}_{2} \mathrm{Et},\left(\mathrm{CH}_{2}\right)_{5} \mathrm{CO}_{2} \mathrm{H} \\
& \mathrm{MY}=\mathrm{NaBF}_{4}, \mathrm{KPF}_{6}, \mathrm{CF}_{3} \mathrm{COONa}, \mathrm{NaNCS}, \mathrm{NaN}(\mathrm{CN})_{2}, \mathrm{NaNO}_{3}
\end{aligned}
$$

Scheme 1. (i) $\mathrm{N}$-alkylation of picoline derivative: conventional preparation, ultrasonic and microwave irradiation conditions. $\mathrm{CP}_{1}$ : $\mathrm{RBr}$, toluene, $80^{\circ} \mathrm{C}, 18 \mathrm{~h}$; US: toluene, $80^{\circ} \mathrm{C}, 5 \mathrm{~h}$; $\mathrm{MW}$ : toluene, $80^{\circ} \mathrm{C}, 15 \mathrm{~min}$. (ii) Anion metathesis using conventional preparation, ultrasonic and microwave irradiation conditions. $\mathrm{CP}$ : MY, acetonitrile, $70^{\circ} \mathrm{C}, 3 \mathrm{~h}$; US: acetonitrile, $70^{\circ} \mathrm{C}, 45 \mathrm{~min}$; $\mathrm{MW}$ : acetonitrile, $70^{\circ} \mathrm{C}, 7 \mathrm{~min}$. 
M. Messali: Conventional versus ultrasound and microwave assisted synthesis: Some new environmentally friendly functionalized picolinium-based ionic liquids with potential antibacterial activity, Acta Pharm. 65 (2015) 253-270.

Table I. Reaction conditions and yields for the synthesis of picolinium-based ionic liquids using conventional preparation and microwave irradiation conditions

\begin{tabular}{|c|c|c|c|c|c|}
\hline \multirow[t]{2}{*}{ Picoline } & \multirow[t]{2}{*}{$\mathrm{RX}$} & \multirow[t]{2}{*}{ IL } & \multicolumn{3}{|c|}{$\begin{array}{l}\text { Yield for } N \text {-alkylation } \\
\text { (first step) (\%) }\end{array}$} \\
\hline & & & $\mathrm{CP}^{\mathrm{a}}$ & $\mathrm{US}^{\mathrm{b}}$ & $\mathrm{MW}^{\mathrm{c}}$ \\
\hline \multirow{2}{*}{ 3-picoline } & $\mathrm{PhO}\left(\mathrm{CH}_{2}\right) 4 \mathrm{Br}$ & 1 & 72 & 79 & 92 \\
\hline & $\mathrm{EtO}_{2} \mathrm{C}\left(\mathrm{CH}_{2}\right)_{5} \mathrm{Br}$ & 2 & 73 & 81 & 92 \\
\hline \multirow{3}{*}{ 4-picoline } & $\mathrm{PhO}\left(\mathrm{CH}_{2}\right)_{4} \mathrm{Br}$ & 3 & 81 & 84 & 93 \\
\hline & $\mathrm{EtO}_{2} \mathrm{C}\left(\mathrm{CH}_{2}\right)_{5} \mathrm{Br}$ & 4 & 79 & 82 & 91 \\
\hline & $\mathrm{HO}_{2} \mathrm{C}\left(\mathrm{CH}_{2}\right)_{5} \mathrm{Br}$ & 5 & 82 & 84 & 94 \\
\hline
\end{tabular}

a Time $18 \mathrm{~h}$, temperature $80^{\circ} \mathrm{C}$, in toluene.

${ }^{\mathrm{b}}$ Time $5 \mathrm{~h}$, temperature $80^{\circ} \mathrm{C}$ in toluene.

${ }^{\mathrm{c}}$ Time $15 \mathrm{~min}$, temperature $80^{\circ} \mathrm{C}$, power $240 \mathrm{~W}$, pressure $2.75 \times 10^{5} \mathrm{~Pa}$.

With the same objective, the microwave assisted preparation of picolinium-based ionic liquids was explored. The reaction conditions were optimized by using either temperature or power control. As mentioned in Table I, the reaction time was reduced to 15 min with a net improvement of isolated yield (92-94\%).

The elemental analysis of all compounds showed that the difference between theoretical and observed mass fractions of carbon, hydrogen and nitrogen was less than $0.3 \%$, which fully confirms the purity of the newly synthesized ILs.

The structure of ILs 1-5 was confirmed by ${ }^{1} \mathrm{H}$ NMR, ${ }^{13} \mathrm{C}$ NMR, IR, LCMS and elemental analysis data. The ${ }^{1} \mathrm{H}$ NMR spectrum showed a singlet around $\delta_{\mathrm{H}} 2.5 \mathrm{ppm}$ corresponding to $\mathrm{CH}_{3}$ protons attached to the pyridinium ring. The characteristic ester protons appeared as a triplet at $\delta 1.11-1.27 \mathrm{ppm}$ and a quartet at $\delta_{\mathrm{H}} 4.03-4.79 \mathrm{ppm}$. The signals at $\delta_{\mathrm{H}}$ 7.67-9.03 ppm were attributed to the pyridinium protons. The phenyl protons of ILs 1 and 3 were been observed at their usual chemical shifts with $\delta_{\mathrm{H}} 6.64-7.11 \mathrm{ppm}$. The singlet at $\delta_{\mathrm{H}} 12.04 \mathrm{ppm}$ in the ${ }^{1} \mathrm{H}$ NMR spectra of compound 5 was attributed to $\mathrm{CO}_{2} \mathrm{H}$.

In ${ }^{13} \mathrm{C}$ NMR spectra of ILs 1-5, all aromatic carbons and $\mathrm{C}=\mathrm{N}$ gave signals between $\delta_{\mathrm{C}}$ 114.3-158.9 ppm. In addition, the carbon of the carbonyl group of ILs $\mathbf{2}, \mathbf{4}$ and 5 appeared around $\delta_{\mathrm{C}} 175.5 \mathrm{ppm} . \mathrm{CH}_{2}$ and $\mathrm{CH}_{3}$ groups were observed at their usual chemical shifts.

The IR spectra of ILs 2, 4 and 5 showed the principal absorption band at $1730 \mathrm{~cm}^{-1}$, indicating the presence of carbonyl group $(\mathrm{C}=\mathrm{O})$ in the molecules, which confirms the success of the $N$-alkylation reaction.

In the second step, a range of anions were introduced by a metathesis reaction using the conventional procedure, ultrasound and microwave irradiation conditions (Scheme 1). The results are given in Table II showing that very good yields were obtained regardless of the procedure. 
M. Messali: Conventional versus ultrasound and microwave assisted synthesis: Some new environmentally friendly functionalized picolinium-based ionic liquids with potential antibacterial activity, Acta Pharm. 65 (2015) 253-270.

Table II. Reaction conditions and yields for the anion metathesis reaction using conventional preparation, ultrasound and microwave irradiation conditions

\begin{tabular}{|c|c|c|c|c|c|c|}
\hline \multirow{2}{*}{ IL } & \multirow{2}{*}{ Cation } & \multirow{2}{*}{$\mathrm{R}$} & \multirow{2}{*}{ MY } & \multicolumn{3}{|c|}{ Yield for anion metathesis (\%) } \\
\hline & & & & $\mathrm{CP}^{\mathrm{a}}$ & $\mathrm{US}^{\mathrm{b}}$ & $\mathrm{MW}^{\mathrm{c}}$ \\
\hline 6 & \multirow{6}{*}{ 3-picolinium } & \multirow{6}{*}{$\mathrm{PhO}\left(\mathrm{CH}_{2}\right)_{4}$} & $\mathrm{NaBF}_{4}$ & 94 & 97 & 98 \\
\hline 7 & & & $\mathrm{KPF}_{6}$ & 93 & 96 & 97 \\
\hline 8 & & & $\mathrm{NaOOCCF}_{3}$ & 95 & 97 & 97 \\
\hline 9 & & & NaNCS & 92 & 96 & 97 \\
\hline 10 & & & $\mathrm{NaN}(\mathrm{CN})_{2}$ & 93 & 95 & 95 \\
\hline 11 & & & $\mathrm{NaNO}_{3}$ & 94 & 97 & 98 \\
\hline 12 & \multirow{6}{*}{ 3-picolinium } & \multirow{6}{*}{$\mathrm{EtO}_{2} \mathrm{C}\left(\mathrm{CH}_{2}\right)_{5}$} & $\mathrm{NaBF}_{4}$ & 94 & 98 & 97 \\
\hline 13 & & & $\mathrm{KPF}_{6}$ & 93 & 97 & 96 \\
\hline 14 & & & $\mathrm{NaOOCCF}_{3}$ & 93 & 97 & 97 \\
\hline 15 & & & NaNCS & 92 & 96 & 96 \\
\hline 16 & & & $\mathrm{NaN}(\mathrm{CN})_{2}$ & 93 & 95 & 95 \\
\hline 17 & & & $\mathrm{NaNO}_{3}$ & 91 & 96 & 97 \\
\hline 18 & \multirow{6}{*}{ 4-picolinium } & \multirow{6}{*}{$\mathrm{PhO}\left(\mathrm{CH}_{2}\right)_{4}$} & $\mathrm{NaBF}_{4}$ & 95 & 98 & 97 \\
\hline 19 & & & $\mathrm{KPF}_{6}$ & 93 & 96 & 96 \\
\hline 20 & & & $\mathrm{NaOOCCF}_{3}$ & 95 & 97 & 95 \\
\hline 21 & & & NaNCS & 94 & 97 & 98 \\
\hline 22 & & & $\mathrm{NaN}(\mathrm{CN})_{2}$ & 94 & 95 & 97 \\
\hline 23 & & & $\mathrm{NaNO}_{3}$ & 94 & 98 & 97 \\
\hline 24 & \multirow{6}{*}{ 4-picolinium } & \multirow{6}{*}{$\mathrm{EtO}_{2} \mathrm{C}\left(\mathrm{CH}_{2}\right)_{5}$} & $\mathrm{NaBF}_{4}$ & 95 & 98 & 96 \\
\hline 25 & & & $\mathrm{KPF}_{6}$ & 95 & 97 & 95 \\
\hline 26 & & & $\mathrm{NaOOCCF}_{3}$ & 92 & 97 & 96 \\
\hline 27 & & & NaNCS & 94 & 96 & 97 \\
\hline 28 & & & $\mathrm{NaN}(\mathrm{CN})_{2}$ & 92 & 95 & 95 \\
\hline 29 & & & $\mathrm{NaNO}_{3}$ & 94 & 96 & 98 \\
\hline 30 & \multirow{6}{*}{ 4-picolinium } & \multirow{6}{*}{$\mathrm{HO}_{2} \mathrm{C}\left(\mathrm{CH}_{2}\right)_{5}$} & $\mathrm{NaBF}_{4}$ & 94 & 96 & 97 \\
\hline 31 & & & $\mathrm{KPF}_{6}$ & 93 & 97 & 97 \\
\hline 32 & & & $\mathrm{NaOOCCF}_{3}$ & 93 & 98 & 95 \\
\hline 33 & & & NaNCS & 92 & 97 & 97 \\
\hline 34 & & & $\mathrm{NaN}(\mathrm{CN})_{2}$ & 93 & 97 & 96 \\
\hline 35 & & & $\mathrm{NaNO}_{3}$ & 92 & 95 & 95 \\
\hline
\end{tabular}

a Time $3 \mathrm{~h}$, temperature $70^{\circ} \mathrm{C}$, in actonitrile.

${ }^{\mathrm{b}}$ Time $45 \mathrm{~min}$, temperature $70^{\circ} \mathrm{C}$ in acetonitrile.

${ }^{\mathrm{c}}$ Time $7 \mathrm{~min}$, temperature $70^{\circ} \mathrm{C}$, power $240 \mathrm{~W}$, pressure $2.75 \times 10^{5} \mathrm{~Pa}$. 
Structural assignments of ILs 6-35 were based on ${ }^{1} \mathrm{H}$ NMR, ${ }^{13} \mathrm{C}$ NMR, IR, LC-MS and elemental analysis data. Furthermore, the ${ }^{11} \mathrm{~B} \mathrm{NMR},{ }^{19} \mathrm{~F} N \mathrm{NM},{ }^{31} \mathrm{P}$ NMR spectra proved without ambiguity the success of metathesis reactions.

The ${ }^{11} \mathrm{~B}$ NMR and ${ }^{19} \mathrm{~F}$ NMR spectra of ILs 6, 12, 18, 24 and 30 contained a singlet around $\delta_{\mathrm{B}}-1$ and $\delta_{\mathrm{F}}-150 \mathrm{ppm}$, respectively, indicating the presence of $\mathrm{BF}_{4}{ }^{-}$. The ${ }^{31} \mathrm{P} \mathrm{NMR}$ and ${ }^{19} \mathrm{~F}$ NMR spectra of ILs 7, 13, 19, 25 and 31 showed a septuplet at $\delta_{\mathrm{P}}-131$ to $-157 \mathrm{ppm}$ related to $\mathrm{PF}_{6}{ }^{-}$. On the other hand, a doublet around $\delta_{\mathrm{F}}-69$ to $-71 \mathrm{ppm}$ confirmed the expected coupling between fluorine and phosphorus. The structure of ILs 8, 14, 20, 26 and 32 was also confirmed from their ${ }^{19} \mathrm{~F}$ NMR spectra by the presence of a singlet at $\delta_{\mathrm{F}}-73 \mathrm{ppm}$ related to $\mathrm{CF}_{3} \mathrm{CO}_{2}^{-}$. All spectroscopic data are detailed in Tables III and IV.

Table III. Physicochemical data for the newly synthesized compounds 1-35

\begin{tabular}{|c|c|c|c|c|}
\hline \multirow{2}{*}{ IL } & \multicolumn{3}{|c|}{ Calcd./found (\%) } & \multirow{2}{*}{ M.p. $\left({ }^{\circ} \mathrm{C}\right)$} \\
\hline & C & $\mathrm{H}$ & $\mathrm{N}$ & \\
\hline 1 & $59.60 / 59.71$ & $6.26 / 6.31$ & $4.35 / 4.42$ & - \\
\hline 2 & $53.17 / 53.22$ & 7.01/7.09 & $4.4 / 4.50$ & - \\
\hline 3 & $59.64 / 59.70$ & $6.26 / 6.33$ & $4.35 / 4.32$ & - \\
\hline 4 & $53.17 / 53.25$ & 7.01/7.11 & $4.43 / 4.49$ & - \\
\hline 5 & $50.01 / 50.07$ & $6.30 / 6.36$ & $4.8 / 4.82$ & 124-125 \\
\hline 6 & $58.39 / 58.47$ & $6.12 / 6.20$ & $4.26 / 4.31$ & - \\
\hline 7 & $49.62 / 49.66$ & $5.20 / 5.23$ & $3.62 / 3.68$ & - \\
\hline 8 & $60.84 / 60.79$ & $5.67 / 5.71$ & $3.94 / 4.01$ & - \\
\hline 9 & $67.97 / 68.03$ & $6.71 / 6.78$ & $9.32 / 9.39$ & - \\
\hline 10 & 70.11/70.17 & $6.54 / 6.60$ & $18.17 / 18.23$ & - \\
\hline 11 & $63.14 / 63.21$ & $6.62 / 6.69$ & $9.20 / 9.16$ & - \\
\hline 12 & $52.04 / 52.11$ & $6.86 / 6.93$ & $4.33 / 4.39$ & - \\
\hline 13 & $44.10 / 44.15$ & $6.86 / 6.93$ & $4.33 / 4.39$ & - \\
\hline 14 & $55.01 / 55.09$ & $6.35 / 6.30$ & $4.01 / 3.97$ & - \\
\hline 15 & $61.19 / 61.27$ & $7.53 / 7.61$ & $9.52 / 9.60$ & - \\
\hline 16 & $63.55 / 63.62$ & $7.33 / 7.30$ & $18.53 / 18.57$ & - \\
\hline 17 & $56.36 / 56.43$ & $7.43 / 7.51$ & $9.39 / 9.45$ & - \\
\hline 18 & $58.39 / 58.30$ & $6.12 / 6.09$ & $4.26 / 4.32$ & - \\
\hline 19 & $49.62 / 49.67$ & $5.20 / 5.28$ & $3.62 / 3.66$ & - \\
\hline 20 & $60.84 / 60.92$ & $5.67 / 5.72$ & $3.94 / 4.01$ & - \\
\hline 21 & $67.97 / 68.02$ & $6.71 / 6.79$ & $9.32 / 9.37$ & - \\
\hline 22 & $70.11 / 70.15$ & $6.54 / 6.59$ & $18.17 / 18.23$ & - \\
\hline 23 & $63.14 / 63.20$ & $6.62 / 6.68$ & $9.20 / 9.26$ & $64-66$ \\
\hline 24 & $52.04 / 52.09$ & $6.86 / 6.92$ & $4.33 / 4.39$ & - \\
\hline
\end{tabular}


M. Messali: Conventional versus ultrasound and microwave assisted synthesis: Some new environmentally friendly functionalized picolinium-based ionic liquids with potential antibacterial activity, Acta Pharm. 65 (2015) 253-270.

Table III. continued

\begin{tabular}{ccccc}
\hline & \multicolumn{3}{c}{ Calcd./found (\%) } & \multirow{2}{*}{ M.p. $\left({ }^{\circ} \mathrm{C}\right)$} \\
\cline { 2 - 4 } IL & $\mathrm{C}$ & $\mathrm{H}$ & $\mathrm{N}$ & - \\
\hline $\mathbf{2 5}$ & $44.10 / 44.18$ & $5.82 / 5.87$ & $3.67 / 3.72$ & - \\
$\mathbf{2 6}$ & $55.01 / 55.08$ & $6.35 / 6.42$ & $4.01 / 4.07$ & - \\
$\mathbf{2 7}$ & $61.19 / 61.13$ & $7.53 / 7.49$ & $9.52 / 9.56$ & - \\
$\mathbf{2 8}$ & $63.55 / 63.61$ & $7.33 / 7.40$ & $18.53 / 18.59$ & - \\
$\mathbf{2 9}$ & $56.36 / 56.43$ & $7.43 / 7.46$ & $9.39 / 9.43$ & $72-74$ \\
$\mathbf{3 0}$ & $48.84 / 48.91$ & $6.15 / 6.22$ & $4.75 / 4.80$ & $57-59$ \\
$\mathbf{3 1}$ & $40.80 / 40.87$ & $5.14 / 5.22$ & $3.97 / 4.05$ & $65-66$ \\
$\mathbf{3 2}$ & $52.34 / 52.39$ & $5.65 / 5.71$ & $4.36 / 4.42$ & $84-86$ \\
$\mathbf{3 3}$ & $58.62 / 58.68$ & $6.81 / 6.87$ & $10.52 / 10.60$ & - \\
$\mathbf{3 4}$ & $61.30 / 61.39$ & $6.61 / 6.69$ & $20.42 / 20.50$ & $110-113$ \\
$\mathbf{3 5}$ & $53.33 / 53.36$ & $6.71 / 6.78$ & $10.36 / 10.43$ & \\
\hline
\end{tabular}

Table IV. ${ }^{1} \mathrm{H},{ }^{13} \mathrm{C},{ }^{19} \mathrm{~F},{ }^{11} \mathrm{~B},{ }^{31} \mathrm{P} N M R$, IR and LCMS spectral data for the newly synthesized ILs 1-35

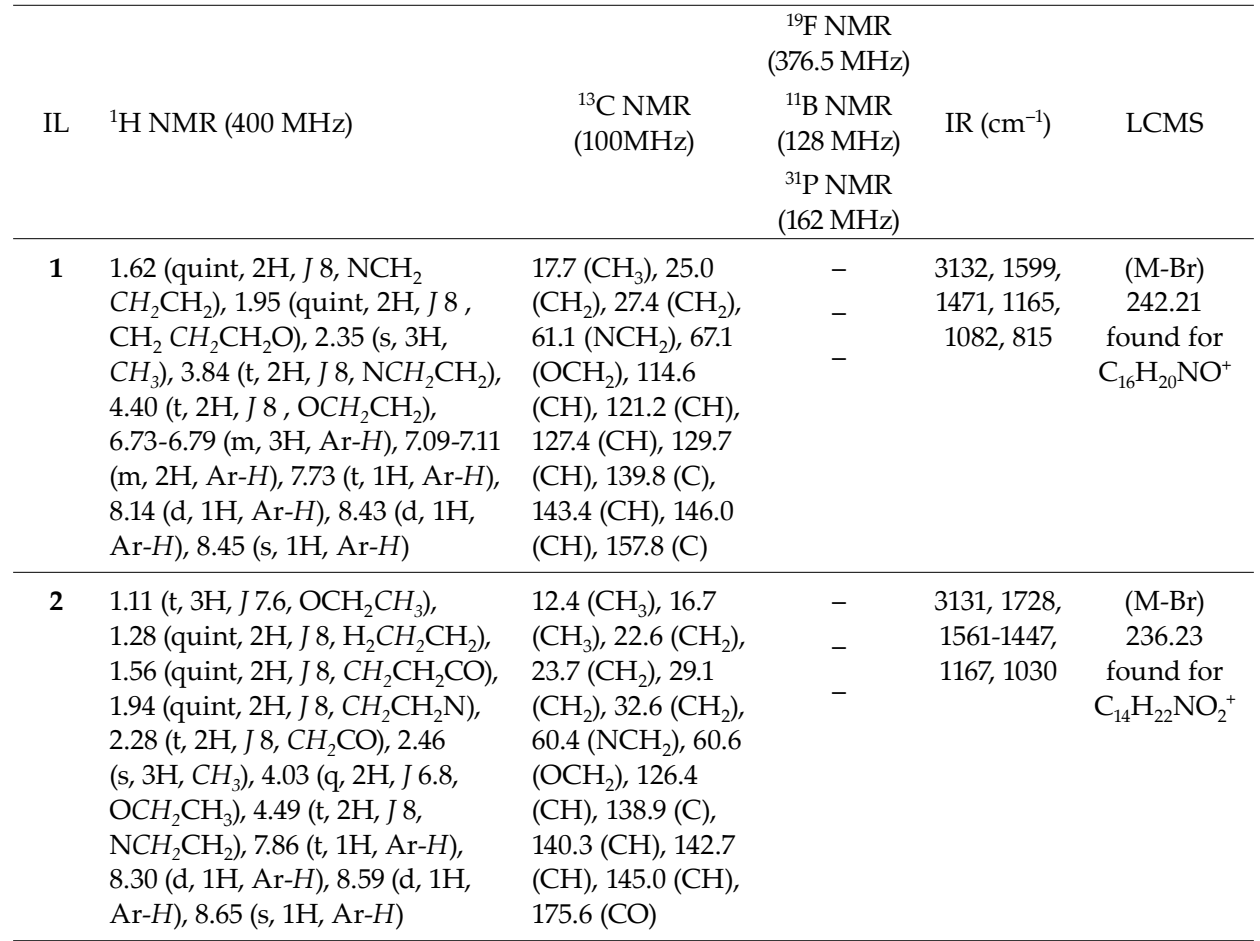


Table IV. continued

\begin{tabular}{|c|c|c|c|c|c|}
\hline 3 & $\begin{array}{l}1.71 \text { (quint, } 2 \mathrm{H}, \mathrm{J} 8, \mathrm{NCH}_{2} \\
\left.\mathrm{CH}_{2} \mathrm{CH}_{2}\right), 2.07 \text { (quint, } 2 \mathrm{H}, J 8, \\
\left.\mathrm{CH}_{2} \mathrm{CH}_{2} \mathrm{CH}_{2} \mathrm{O}\right), 2.41(\mathrm{~s}, 3 \mathrm{H}, \\
\left.\mathrm{CH}_{3}\right), 3.80\left(\mathrm{t}, 2 \mathrm{H}, J 8, \mathrm{NCH}_{2} \mathrm{CH}_{2}\right) \\
4.79\left(\mathrm{t}, 2 \mathrm{H}, J 8, \mathrm{OCH} \mathrm{CH}_{2}\right) \\
6.64-6.74(\mathrm{~m}, 3 \mathrm{H}, \mathrm{Ar}-\mathrm{H}), 7.03-7.07 \\
(\mathrm{~m}, 2 \mathrm{H}, \mathrm{Ar}-\mathrm{H}), 7.67(\mathrm{~d}, 2 \mathrm{H}, \mathrm{Ar}-\mathrm{H}), \\
9.23(\mathrm{~d}, 2 \mathrm{H}, \mathrm{Ar}-\mathrm{H})\end{array}$ & $\begin{array}{l}22.0\left(\mathrm{CH}_{3}\right), 25.5 \\
\left(\mathrm{CH}_{2}\right), 28.6\left(\mathrm{CH}_{2}\right) \\
60.3\left(\mathrm{NCH}_{2}\right), 66.7 \\
\left(\mathrm{OCH}_{2}\right), 114.3 \\
(\mathrm{CH}), 120.7(\mathrm{CH}) \\
128.7(\mathrm{CH}), 129.3 \\
(\mathrm{CH}), 144.1(\mathrm{CH}) \\
158.4(\mathrm{C}), 158.8(\mathrm{C})\end{array}$ & $\begin{array}{l}- \\
- \\
-\end{array}$ & $\begin{array}{c}3130,1597 \\
1469,1163 \\
1080,813\end{array}$ & $\begin{array}{c}(\mathrm{M}-\mathrm{Br}) \\
242.21 \\
\text { found for } \\
\mathrm{C}_{16} \mathrm{H}_{20} \mathrm{NO}^{+}\end{array}$ \\
\hline 4 & $\begin{array}{l}1.15\left(\mathrm{t}, 3 \mathrm{H}, J \mathrm{~J} .6, \mathrm{OCH}_{2} \mathrm{CH}_{3}\right), 1.28 \\
\left.\text { (quint, } 2 \mathrm{H}, \mathrm{J} 8, \mathrm{CH}_{2} \mathrm{CH}_{2} \mathrm{CH}_{2}\right), \\
\left.1.57 \text { (quint, } 2 \mathrm{H}, J 8, \mathrm{CH}_{2} \mathrm{CH}_{2} \mathrm{CO}\right), \\
\left.1.95 \text { (quint, } 2 \mathrm{H}, J 8, \mathrm{CH}_{2} \mathrm{CH}_{2} \mathrm{~N}\right), \\
2.30\left(\mathrm{t}, 2 \mathrm{H}, J 8, \mathrm{CH}_{2} \mathrm{CO}\right), 2.59(\mathrm{~s}, \\
\left.3 \mathrm{H}, \mathrm{CH}_{3}\right), 4.04(\mathrm{q}, 2 \mathrm{H}, J 6.8, \\
\left.\mathrm{OCH}_{2} \mathrm{CH}_{3}\right), 4.49(\mathrm{t}, 2 \mathrm{H}, J 8, \\
\left.\mathrm{NCH}_{2} \mathrm{CH}_{2}\right), 7.84(\mathrm{~d}, 2 \mathrm{H}, \mathrm{Ar}-\mathrm{H}), \\
8.62(\mathrm{~d}, 2 \mathrm{H}, \mathrm{Ar}-\mathrm{H})\end{array}$ & $\begin{array}{l}12.4\left(\mathrm{CH}_{3}\right), 20.3 \\
\left(\mathrm{CH}_{3}\right), 22.6\left(\mathrm{CH}_{2}\right), \\
23.6\left(\mathrm{CH}_{2}\right), 29.0 \\
\left(\mathrm{CH}_{2}\right), 32.6\left(\mathrm{CH}_{2}\right) \\
59.6\left(\mathrm{NCH}_{2}\right), 60.5 \\
\left(\mathrm{OCH}_{2}\right), 127.6 \\
(\mathrm{CH}), 142.0(\mathrm{CH}) \\
145.9(\mathrm{CH}), 158.9 \\
(\mathrm{C}), 175.4(\mathrm{CO})\end{array}$ & $\begin{array}{l}- \\
- \\
-\end{array}$ & $\begin{array}{c}3132,1730 \\
1560,1448 \\
1166,1031\end{array}$ & $\begin{array}{c}\text { (M-Br) } \\
236.23 \\
\text { found for } \\
\mathrm{C}_{14} \mathrm{H}_{22} \mathrm{NO}_{2}^{+}\end{array}$ \\
\hline 5 & $\begin{array}{l}\delta 1.27 \text { (quint, 2H, } J \text { 8, } \\
\mathrm{CH}_{2} \mathrm{CH}_{2} \mathrm{CH}_{2} \text { ), } 1.53 \text { (quint, } 2 \mathrm{H}, \\
J 8, \mathrm{CH}_{2} \mathrm{CH}_{2} \mathrm{CO} \text { ), } 1.89 \text { (quint, } \\
\left.2 \mathrm{H}, \mathrm{J} 8, \mathrm{CH}_{2} \mathrm{CH}_{2} \mathrm{~N}\right), 2.22(\mathrm{t}, 2 \mathrm{H}, \\
\left.J 8, \mathrm{CH}_{2} \mathrm{CO}\right), 2.53\left(\mathrm{~s}, 3 \mathrm{H}, \mathrm{CH}_{3}\right), \\
4.58\left(\mathrm{t}, 2 \mathrm{H}, \mathrm{J} 6.8, \mathrm{NCH}_{2} \mathrm{CH}_{2}\right), \\
8.03(\mathrm{~d}, 2 \mathrm{H}, \mathrm{Ar}-\mathrm{H}), 9.03(\mathrm{~d}, 2 \mathrm{H}, \\
\text { Ar- } \mathrm{H}), 12.04\left(\mathrm{~s}, 1 \mathrm{H}, \mathrm{CO}_{2} \mathrm{H}\right)\end{array}$ & $\begin{array}{l}20.1\left(\mathrm{CH}_{3}\right), 22.5 \\
\left(\mathrm{CH}_{2}\right), 23.6\left(\mathrm{CH}_{2}\right) \\
29.0\left(\mathrm{CH}_{2}\right), 32.0 \\
\left(\mathrm{CH}_{2}\right), 58.3 \\
\left(\mathrm{NCH}_{2}\right), 127.1 \\
(\mathrm{CH}), 142.5(\mathrm{CH}) \\
157.5(\mathrm{C}), 173.0 \\
(\mathrm{CO})\end{array}$ & $\begin{array}{l}- \\
- \\
-\end{array}$ & $\begin{array}{c}3256,3130 \\
1728 \\
1559-1449 \\
1168,1032\end{array}$ & $\begin{array}{c}\text { (M-Br) } \\
208.22 \\
\text { found for } \\
\mathrm{C}_{12} \mathrm{H}_{18} \mathrm{NO}_{2}^{+}\end{array}$ \\
\hline 6 & $\begin{array}{l}1.80 \text { (quint, } 2 \mathrm{H}, \mathrm{J} 8, \mathrm{NCH}_{2} \\
\left.\mathrm{CH}_{2} \mathrm{CH}_{2}\right), 2.15 \text { (quint, } 2 \mathrm{H}, J 8, \\
\left.\mathrm{CH}_{2} \mathrm{CH}_{2} \mathrm{CH}_{2} \mathrm{O}\right), 2.49(\mathrm{~s}, 3 \mathrm{H}, \\
\left.\mathrm{CH}_{3}\right), 3.92\left(\mathrm{t}, 2 \mathrm{H}, \mathrm{J} 8, \mathrm{NCH}_{2} \mathrm{CH}_{2}\right), \\
4.61\left(\mathrm{t}, 2 \mathrm{H}, \mathrm{J} 8, \mathrm{OCH}_{2} \mathrm{CH}_{2}\right) \\
\text { 6.79-6.89 (m, 3H, Ar- } \mathrm{H}), \\
7.18-7.22(\mathrm{~m}, 2 \mathrm{H}, \mathrm{Ar}-\mathrm{H}), 7.84(\mathrm{t}, \\
1 \mathrm{H}, \mathrm{Ar}-\mathrm{H}), 8.14(\mathrm{~d}, 1 \mathrm{H}, \mathrm{Ar}-\mathrm{H}), \\
8.63(\mathrm{~d}, 1 \mathrm{H}, \mathrm{Ar}-\mathrm{H}), 8.68(\mathrm{~s}, 1 \mathrm{H}, \\
\text { Ar- } \mathrm{H})\end{array}$ & $\begin{array}{l}16.5\left(\mathrm{CH}_{3}\right), 23.8 \\
\left(\mathrm{CH}_{2}\right), 26.7\left(\mathrm{CH}_{2}\right), \\
59.8\left(\mathrm{NCH}_{2}\right), 64.9 \\
(\mathrm{OCH}), 112.6 \\
(\mathrm{CH}), 119.0(\mathrm{CH}), \\
126.0(\mathrm{CH}), 127.7 \\
(\mathrm{CH}), 138.3(\mathrm{C}), \\
139.7(\mathrm{CH}), \\
142.2(\mathrm{CH}), \\
144.1(\mathrm{CH}), \\
156.7(\mathrm{C})\end{array}$ & $\begin{array}{c}-151.08(\mathrm{~s}) \\
-0.95(\mathrm{~s}) \\
-\end{array}$ & $\begin{array}{c}3131,1598 \\
1471,1163 \\
1081,816\end{array}$ & $\begin{array}{c}\left(\mathrm{M}-\mathrm{BF}_{4}\right) \\
242.20 \\
\text { found for } \\
\mathrm{C}_{16} \mathrm{H}_{20} \mathrm{NO}^{+}\end{array}$ \\
\hline I & $\begin{array}{l}1.76 \text { (quint, } 2 \mathrm{H}, \mathrm{J} 8, \mathrm{NCH}_{2} \\
\left.\mathrm{CH}_{2} \mathrm{CH}_{2}\right), 2.10 \text { (quint, } 2 \mathrm{H}, J 8, \\
\left.\mathrm{CH}_{2} \mathrm{CH}_{2} \mathrm{CH}_{2} \mathrm{O}\right), 2.51(\mathrm{~s}, 3 \mathrm{H}, \\
\left.\mathrm{CH}_{3}\right), 4.01\left(\mathrm{t}, 2 \mathrm{H}, \mathrm{J} 8, \mathrm{NCH}_{2} \mathrm{CH}_{2}\right), \\
4.54\left(\mathrm{t}, 2 \mathrm{H}, \mathrm{J} 8, \mathrm{OCH} \mathrm{CH}_{2}\right), \\
\text { 6.91-6.95 (m, 3H, Ar-H), } \\
7.27-7.33(\mathrm{~m}, 2 \mathrm{H}, \mathrm{Ar}-\mathrm{H}), 8.05(\mathrm{t}, \\
\text { 1H, Ar- } \mathrm{H}), 8.44(\mathrm{~d}, 1 \mathrm{H}, \mathrm{Ar}-\mathrm{H}), \\
8.94(\mathrm{~d}, 1 \mathrm{H}, \mathrm{Ar}-\mathrm{H}), 9.00(\mathrm{~s}, 1 \mathrm{H}, \\
\text { Ar- } \mathrm{H})\end{array}$ & $\begin{array}{l}16.7\left(\mathrm{CH}_{3}\right), 24.2 \\
\left(\mathrm{CH}_{2}\right), 26.6\left(\mathrm{CH}_{2}\right), \\
59.4\left(\mathrm{NCH}_{2}\right), 65.4 \\
\left(\mathrm{OCH}_{2}\right), 113.3 \\
(\mathrm{CH}), 119.5(\mathrm{CH}), \\
126.3(\mathrm{CH}), 128.4 \\
(\mathrm{CH}), 137.8(\mathrm{C}), \\
140.8(\mathrm{CH}), 143.1 \\
(\mathrm{CH}), 144.7(\mathrm{CH}), \\
157.3(\mathrm{C})\end{array}$ & $\begin{array}{c}-69.25 \\
-71.14(\mathrm{~d}) \\
- \\
-131.00 \\
-157.35 \\
\text { (sept) }\end{array}$ & $\begin{array}{c}3133,1599 \\
1473,1163 \\
1081,815\end{array}$ & $\begin{array}{c}\left(\mathrm{M}-\mathrm{PF}_{6}\right) \\
242.23 \\
\text { found for } \\
\mathrm{C}_{16} \mathrm{H}_{20} \mathrm{NO}^{+}\end{array}$ \\
\hline
\end{tabular}


Table IV. continued

\begin{tabular}{|c|c|c|c|c|c|}
\hline 8 & $\begin{array}{l}1.79 \text { (quint, } 2 \mathrm{H}, \mathrm{J} 8, \mathrm{NCH}_{2} \\
\left.\mathrm{CH}_{2} \mathrm{CH}_{2}\right), 2.17 \text { (quint, } 2 \mathrm{H}, J 8, \\
\left.\mathrm{CH}_{2} \mathrm{CH}_{2} \mathrm{CH}_{2} \mathrm{O}\right), 2.53(\mathrm{~s}, 3 \mathrm{H}, \\
\left.\mathrm{CH}_{3}\right), 4.01\left(\mathrm{t}, 2 \mathrm{H}, J 8, \mathrm{NCH}_{2} \mathrm{CH}_{2}\right), \\
4.75\left(\mathrm{t}, 2 \mathrm{H}, \mathrm{J} 8, \mathrm{OCH}_{2} \mathrm{CH}_{2}\right) \\
6.89-6.93(\mathrm{~m}, 3 \mathrm{H}, \mathrm{Ar}-\mathrm{H}), \\
7.24-7.28(\mathrm{~m}, 2 \mathrm{H}, \mathrm{Ar}-\mathrm{H}), 8.07(\mathrm{t}, \\
1 \mathrm{H}, \mathrm{Ar}-\mathrm{H}), 8.47(\mathrm{~d}, 1 \mathrm{H}, \mathrm{Ar}-\mathrm{H}), \\
9.12(\mathrm{~d}, 1 \mathrm{H}, \mathrm{Ar}-\mathrm{H}), 9.23(\mathrm{~s}, 1 \mathrm{H}, \\
\text { Ar- } \mathrm{H})\end{array}$ & $\begin{array}{l}16.6\left(\mathrm{CH}_{3}\right), 24.1 \\
\left(\mathrm{CH}_{2}\right), 26.6\left(\mathrm{CH}_{2}\right), \\
59.1\left(\mathrm{NCH}_{2}\right), 65.3 \\
(\mathrm{OCH}), 113.1 \\
(\mathrm{CH}), 119.2(\mathrm{CH}), \\
126.2(\mathrm{CH}), 128.1 \\
(\mathrm{CH}), 137.6(\mathrm{C}) \\
140.9(\mathrm{CH}), 143.2 \\
(\mathrm{CH}), 144.6(\mathrm{CH}), \\
157.2(\mathrm{C})\end{array}$ & $\begin{array}{c}-73.92(\mathrm{~s}) \\
- \\
-\end{array}$ & $\begin{array}{c}3130,1600 \\
1471,1165 \\
1084,815\end{array}$ & $\begin{array}{c}\left(\mathrm{M}-\mathrm{CF}_{3} \mathrm{CO}_{2}\right) \\
242.22 \\
\text { found for } \\
\mathrm{C}_{16} \mathrm{H}_{20} \mathrm{NO}^{+}\end{array}$ \\
\hline 9 & $\begin{array}{l}1.85 \text { (quint, } 2 \mathrm{H}, \mathrm{J} 8, \mathrm{NCH}_{2} \\
\left.\mathrm{CH}_{2} \mathrm{CH}_{2}\right), 2.20 \text { (quint, } 2 \mathrm{H}_{1} \mathrm{~J} 8, \\
\left.\mathrm{CH}_{2} \mathrm{CH}_{2} \mathrm{CH}_{2} \mathrm{O}\right), 2.52(\mathrm{~s}, 3 \mathrm{H}, \\
\left.\mathrm{CH}_{3}\right), 3.94\left(\mathrm{t}, 2 \mathrm{H}, \mathrm{J} 8, \mathrm{NCH}_{2} \mathrm{CH}_{2}\right), \\
4.75\left(\mathrm{t}, 2 \mathrm{H}, \mathrm{J} 8, \mathrm{OCH} \mathrm{CH}_{2}\right), \\
6.78-6.85(\mathrm{~m}, 3 \mathrm{H}, \mathrm{Ar}-\mathrm{H}), \\
7.15-7.19(\mathrm{~m}, 2 \mathrm{H}, \mathrm{Ar}-\mathrm{H}), 7.92(\mathrm{t}, \\
1 \mathrm{H}, \mathrm{Ar}-\mathrm{H}), 8.20(\mathrm{~d}, 1 \mathrm{H}, \mathrm{Ar}-\mathrm{H}), \\
8.82(\mathrm{~d}, 1 \mathrm{H}, \mathrm{Ar}-\mathrm{H}), 8.85(\mathrm{~s}, 1 \mathrm{H}, \\
\text { Ar- } \mathrm{H})\end{array}$ & $\begin{array}{l}16.8\left(\mathrm{CH}_{3}\right), 23.9 \\
\left(\mathrm{CH}_{2}\right), 26.8\left(\mathrm{CH}_{2}\right), \\
59.9\left(\mathrm{NCH}_{2}\right), 64.9 \\
\left(\mathrm{OCH}_{2}\right), 112.6 \\
(\mathrm{CH}), 118.9(\mathrm{CH}), \\
126.1(\mathrm{CH}), 127.6 \\
(\mathrm{CH}), 138.1(\mathrm{C}), \\
139.9(\mathrm{CH}), 142.2 \\
(\mathrm{CH}), 144.0(\mathrm{CH}), \\
156.6(\mathrm{C})\end{array}$ & - & $\begin{array}{c}3131,1599, \\
1470,1164 \\
1081,815\end{array}$ & $\begin{array}{c}\text { (M-NCS) } \\
242.22 \\
\text { found for } \\
\mathrm{C}_{16} \mathrm{H}_{20} \mathrm{NO}^{+}\end{array}$ \\
\hline 10 & $\begin{array}{l}1.75 \text { (quint, } 2 \mathrm{H}, \mathrm{J} 8, \mathrm{NCH}_{2} \\
\left.\mathrm{CH}_{2} \mathrm{CH}_{2}\right), 2.12 \text { (quint, } 2 \mathrm{H}, J 8, \\
\left.\mathrm{CH}_{2} \mathrm{CH}_{2} \mathrm{CH}_{2} \mathrm{O}\right), 2.45(\mathrm{~s}, 3 \mathrm{H}, \\
\left.\mathrm{CH}_{3}\right), 3.87\left(\mathrm{t}, 2 \mathrm{H}, J 8, \mathrm{NCH}_{2} \mathrm{CH}_{2}\right), \\
4.70\left(\mathrm{t}, 2 \mathrm{H}, \mathrm{J} 8, \mathrm{OCH}_{2} \mathrm{CH}_{2}\right), \\
\text { 6.71-6.79 (m, 3H, Ar- } \mathrm{H}), \\
7.09-7.13(\mathrm{~m}, 2 \mathrm{H}, \mathrm{Ar}-\mathrm{H}), 7.80(\mathrm{t}, \\
1 \mathrm{H}, \mathrm{Ar}-\mathrm{H}), 8.10(\mathrm{~d}, 1 \mathrm{H}, \mathrm{Ar}-\mathrm{H}), \\
8.89(\mathrm{~d}, 1 \mathrm{H}, \mathrm{Ar}-\mathrm{H}), 8.98(\mathrm{~s}, 1 \mathrm{H}, \\
\text { Ar- } \mathrm{H})\end{array}$ & $\begin{array}{l}16.7\left(\mathrm{CH}_{3}\right), 23.8 \\
\left(\mathrm{CH}_{2}\right), 26.9\left(\mathrm{CH}_{2}\right), \\
59.5\left(\mathrm{NCH}_{2}\right), 64.9 \\
\left(\mathrm{OCH}_{2}\right), 112.5 \\
(\mathrm{CH}), 119.0(\mathrm{CH}), \\
125.9(\mathrm{CH}), 127.6 \\
(\mathrm{CH}), 138.1(\mathrm{C}), \\
139.9(\mathrm{CH}), 142.3 \\
(\mathrm{CH}), 144.0(\mathrm{CH}), \\
156.6(\mathrm{C})\end{array}$ & $\begin{array}{l}- \\
- \\
-\end{array}$ & $\begin{array}{c}3132,1601 \\
1472,1167 \\
1082,815\end{array}$ & $\begin{array}{c}\left(\mathrm{M}-\mathrm{N}(\mathrm{CN})_{2}\right) \\
242.21 \\
\text { found for } \\
\mathrm{C}_{16} \mathrm{H}_{20} \mathrm{NO}^{+}\end{array}$ \\
\hline 1 & $\begin{array}{l}1.62 \text { (quint, } 2 \mathrm{H}, J 8, \mathrm{NCH}_{2} \\
\left.\mathrm{CH}_{2} \mathrm{CH}_{2}\right), 1.99 \text { (quint, } 2 \mathrm{H}, J 8, \\
\left.\mathrm{CH}_{2} \mathrm{CH}_{2} \mathrm{CH}_{2} \mathrm{O}\right), 2.31(\mathrm{~s}, 3 \mathrm{H}, \\
\left.\mathrm{CH}_{3}\right), 3.71\left(\mathrm{t}, 2 \mathrm{H}, J 8, \mathrm{NCH}_{2} \mathrm{CH}_{2}\right), \\
4.71\left(\mathrm{t}, 2 \mathrm{H}, J 8, \mathrm{OCH}_{2} \mathrm{CH}_{2}\right), \\
6.64-6.75(\mathrm{~m}, 3 \mathrm{H}, \mathrm{Ar}-\mathrm{H}), \\
\text { 6.93-6.97 (m, 2H, Ar- } \mathrm{H}), 7.71(\mathrm{t}, \\
1 \mathrm{H}, \mathrm{Ar}-\mathrm{H}), 7.97(\mathrm{~d}, 1 \mathrm{H}, \mathrm{Ar}-\mathrm{H}), \\
9.05(\mathrm{~d}, 1 \mathrm{H}, \mathrm{Ar}-\mathrm{H}), 9.21(\mathrm{~s}, 1 \mathrm{H}, \\
\text { Ar- } \mathrm{H})\end{array}$ & $\begin{array}{l}16.5\left(\mathrm{CH}_{3}\right), 23.7 \\
\left(\mathrm{CH}_{2}\right), 26.8\left(\mathrm{CH}_{2}\right), \\
58.9\left(\mathrm{NCH}_{2}\right), 64.8 \\
\left(\mathrm{OCH}_{2}\right), 112.4 \\
(\mathrm{CH}), 118.7(\mathrm{CH}), \\
125.8(\mathrm{CH}), 127.4 \\
(\mathrm{CH}), 137.6(\mathrm{C}), \\
140.2(\mathrm{CH}), 142.5 \\
(\mathrm{CH}), 143.8(\mathrm{CH}), \\
156.5(\mathrm{C})\end{array}$ & - & $\begin{array}{c}3132,1598 \\
1470,1162 \\
1080,813\end{array}$ & $\begin{array}{c}\left(\mathrm{M}-\mathrm{NO}_{3}\right) \\
242.21 \\
\text { found for } \\
\mathrm{C}_{16} \mathrm{H}_{20} \mathrm{NO}^{+}\end{array}$ \\
\hline
\end{tabular}


Table IV. continued

\begin{tabular}{|c|c|c|c|c|c|}
\hline 12 & 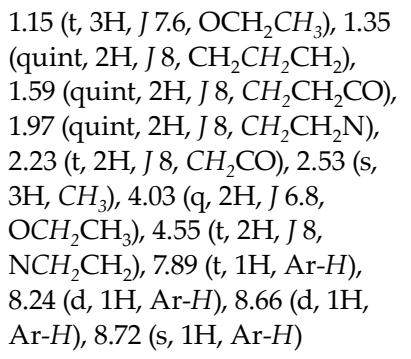 & $\begin{array}{l}12.4\left(\mathrm{CH}_{3}\right), 16.6 \\
\left(\mathrm{CH}_{3}\right), 22.2\left(\mathrm{CH}_{2}\right), \\
23.5\left(\mathrm{CH}_{2}\right), 29.3 \\
\left(\mathrm{CH}_{2}\right), 31.9\left(\mathrm{CH}_{2}\right), \\
58.5\left(\mathrm{NCH}_{2}\right), 59.9 \\
\left(\mathrm{OCH}_{2}\right), 126.1 \\
(\mathrm{CH}), 138.3(\mathrm{C}), \\
139.9(\mathrm{CH}), 142.4 \\
(\mathrm{CH}), 144.2(\mathrm{CH}), \\
171.6(\mathrm{CO})\end{array}$ & $\begin{array}{c}-151.44(\mathrm{~s}) \\
-1.06(\mathrm{~s}) \\
-\end{array}$ & $\begin{array}{c}3131,1728, \\
1559,1449, \\
1167,1032\end{array}$ & $\begin{array}{c}\left(\mathrm{M}-\mathrm{BF}_{4}\right) \\
236.24 \\
\text { found for } \\
\mathrm{C}_{14} \mathrm{H}_{22} \mathrm{NO}_{2}^{+}\end{array}$ \\
\hline 13 & $\begin{array}{l}1.17\left(\mathrm{t}, 3 \mathrm{H}, J \mathrm{~J} .6, \mathrm{OCH}_{2} \mathrm{CH}_{3}\right), 1.34 \\
\text { (quint, 2H, } \mathrm{J}, \mathrm{CH}_{2} \mathrm{CH}_{2} \mathrm{CH}_{2} \text { ), } \\
\left.1.58 \text { (quint, } 2 \mathrm{H}, J 8, \mathrm{CH}_{2} \mathrm{CH}_{2} \mathrm{CO}\right), \\
1.95 \text { (quint, } 2 \mathrm{H}, \mathrm{J} 8, \mathrm{CH}_{2} \mathrm{CH}_{2} \mathrm{~N} \text { ), } \\
2.23\left(\mathrm{t}, 2 \mathrm{H}, J 8, \mathrm{CH}_{2} \mathrm{CO}\right), 2.55(\mathrm{~s}, \\
\left.3 \mathrm{H}, \mathrm{CH}_{3}\right), 4.02(\mathrm{q}, 2 \mathrm{H}, J 6.8, \\
\left.\mathrm{OCH}_{2} \mathrm{CH}_{3}\right), 4.46(\mathrm{t}, 2 \mathrm{H}, J 8, \\
\left.\mathrm{NCH}_{2} \mathrm{CH}_{2}\right), 7.84(\mathrm{t}, 1 \mathrm{H}, \mathrm{Ar}-\mathrm{H}), \\
8.23(\mathrm{~d}, 1 \mathrm{H}, \mathrm{Ar}-\mathrm{H}), 8.49(\mathrm{~d}, 1 \mathrm{H}, \\
\text { Ar- } \mathrm{H}), 8.53(\mathrm{~s}, 1 \mathrm{H}, \mathrm{Ar}-\mathrm{H})\end{array}$ & $\begin{array}{l}12.5\left(\mathrm{CH}_{3}\right), 16.6 \\
\left(\mathrm{CH}_{3}\right), 22.3\left(\mathrm{CH}_{2}\right), \\
23.6\left(\mathrm{CH}_{2}\right), 29.3 \\
\left(\mathrm{CH}_{2}\right), 32.0\left(\mathrm{CH}_{2}\right), \\
58.6\left(\mathrm{NCH}_{2}\right), 60.1 \\
\left(\mathrm{OCH}_{2}\right), 126.2 \\
(\mathrm{CH}), 138.6(\mathrm{C}), \\
139.6(\mathrm{CH}), 142.2 \\
(\mathrm{CH}), 144.5(\mathrm{CH}), \\
171.7(\mathrm{CO})\end{array}$ & $\begin{array}{c}-71.31 \\
-73.20(\mathrm{~d}) \\
- \\
-131.31 \\
-157.68 \\
\text { (sept) }\end{array}$ & $\begin{array}{l}3133,1729, \\
1559,1450, \\
1166,1030\end{array}$ & $\begin{array}{c}\left(\mathrm{M}-\mathrm{PF}_{6}\right) \\
236.20 \\
\text { found for } \\
\mathrm{C}_{14} \mathrm{H}_{22} \mathrm{NO}_{2}^{+}\end{array}$ \\
\hline 14 & $\begin{array}{l}1.13\left(\mathrm{t}, 3 \mathrm{H}, J \mathrm{~J} .6, \mathrm{OCH}_{2} \mathrm{CH}_{3}\right), 1.32 \\
\text { (quint, 2H, } \mathrm{J}, \mathrm{CH}_{2} \mathrm{CH}_{2} \mathrm{CH}_{2} \text { ), } \\
1.58 \text { (quint, } 2 \mathrm{H}, J 8, \mathrm{CH}_{2} \mathrm{CH}_{2} \mathrm{CO} \text { ), } \\
1.95 \text { (quint, 2H, J } 8, \mathrm{CH}_{2} \mathrm{CH}_{2} \mathrm{~N} \text { ), } \\
2.22\left(\mathrm{t}, 2 \mathrm{H}, J 8, \mathrm{CH}_{2} \mathrm{CO}\right), 2.54(\mathrm{~s}, \\
\left.3 \mathrm{H}, \mathrm{CH}_{3}\right), 4.01(\mathrm{q}, 2 \mathrm{H}, J 6.8, \\
\left.\mathrm{OCH}_{2} \mathrm{CH}_{3}\right), 4.74(\mathrm{t}, 2 \mathrm{H}, J 8, \\
\left.\mathrm{NCH}_{2} \mathrm{CH}_{2}\right), 7.93(\mathrm{t}, 1 \mathrm{H}, \mathrm{Ar}-\mathrm{H}), \\
8.21(\mathrm{~d}, 1 \mathrm{H}, \mathrm{Ar}-\mathrm{H}), 9.11(\mathrm{~d}, 1 \mathrm{H}, \\
\text { Ar- } \mathrm{H}), 9.22(\mathrm{~s}, 1 \mathrm{H}, \mathrm{Ar}-\mathrm{H})\end{array}$ & $\begin{array}{l}12.2\left(\mathrm{CH}_{3}\right), 16.5 \\
\left(\mathrm{CH}_{3}\right), 22.1\left(\mathrm{CH}_{2}\right), \\
23.3\left(\mathrm{CH}_{2}\right), 29.5 \\
\left(\mathrm{CH}_{2}\right), 31.8\left(\mathrm{CH}_{2}\right), \\
58.5\left(\mathrm{NCH}_{2}\right), 59.4 \\
\left(\mathrm{OCH}_{2}\right), 126.0 \\
(\mathrm{CH}), 137.9(\mathrm{C}), \\
140.4(\mathrm{CH}), 142.7 \\
(\mathrm{CH}), 143.8(\mathrm{CH}), \\
171.6(\mathrm{CO})\end{array}$ & $\begin{array}{c}-75.34(\mathrm{~s}) \\
- \\
-\end{array}$ & $\begin{array}{c}3132,1730, \\
1558,1448, \\
1167,1032\end{array}$ & $\begin{array}{c}\left(\mathrm{M}-\mathrm{CF}_{3} \mathrm{CO}_{2}\right) \\
236.22 \\
\text { found for } \\
\mathrm{C}_{14} \mathrm{H}_{22} \mathrm{NO}_{2}^{+}\end{array}$ \\
\hline 15 & 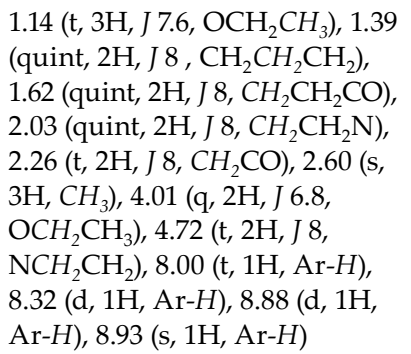 & $\begin{array}{l}12.2\left(\mathrm{CH}_{3}\right), 16.7 \\
\left(\mathrm{CH}_{3}\right), 22.0\left(\mathrm{CH}_{2}\right), \\
23.3\left(\mathrm{CH}_{2}\right), 29.3 \\
\left(\mathrm{CH}_{2}\right), 31.7\left(\mathrm{CH}_{2}\right), \\
58.3\left(\mathrm{NCH}_{2}\right), 59.5 \\
\left(\mathrm{OCH}_{2}\right), 125.9 \\
(\mathrm{CH}), 138.1(\mathrm{C}), \\
139.9(\mathrm{CH}), 142.3 \\
(\mathrm{CH}), 144.1(\mathrm{CH}), \\
171.3(\mathrm{CO})\end{array}$ & $\begin{array}{l}- \\
- \\
-\end{array}$ & $\begin{array}{l}3132,1729, \\
1560,1449, \\
1168,1034\end{array}$ & $\begin{array}{c}\text { (M-NCS) } \\
236.21 \\
\text { found for } \\
\mathrm{C}_{14} \mathrm{H}_{22} \mathrm{NO}_{2}^{+}\end{array}$ \\
\hline
\end{tabular}


Table IV. continued

\begin{tabular}{|c|c|c|c|c|c|}
\hline 16 & $\begin{array}{l}1.01\left(\mathrm{t}, 3 \mathrm{H}, J \mathrm{~J} .6, \mathrm{OCH}_{2} \mathrm{CH}_{3}\right), 1.20 \\
\text { (quint, } 2 \mathrm{H}, J 8, \mathrm{CH}_{2} \mathrm{CH}_{2} \mathrm{CH}_{2} \text { ), } \\
1.44 \text { (quint, } 2 \mathrm{H}, J \mathrm{C}, \mathrm{CH}_{2} \mathrm{CH}_{2} \mathrm{CO} \text { ), } \\
\left.1.81 \text { (quint, } 2 \mathrm{H}, J 8, \mathrm{CH}_{2} \mathrm{CH}_{2} \mathrm{~N}\right), \\
2.07\left(\mathrm{t}, 2 \mathrm{H}, J 8, \mathrm{CH}_{2} \mathrm{CO}\right), 2.42(\mathrm{~s}, \\
\left.3 \mathrm{H}, \mathrm{CH}_{3}\right), 3.84(\mathrm{q}, 2 \mathrm{H}, J 6.8 \\
\left.\mathrm{OCH}_{2} \mathrm{CH}_{3}\right), 4.54(\mathrm{t}, 2 \mathrm{H}, J 8, \\
\left.\mathrm{NCH}_{2} \mathrm{CH}_{2}\right), 7.80(\mathrm{t}, 1 \mathrm{H}, \mathrm{Ar}-\mathrm{H}), \\
8.15(\mathrm{~d}, 1 \mathrm{H}, \mathrm{Ar}-\mathrm{H}), 8.82(\mathrm{~d}, 1 \mathrm{H}, \\
\mathrm{Ar}-\mathrm{H}), 8.92(\mathrm{~s}, 1 \mathrm{H}, \mathrm{Ar}-\mathrm{H})\end{array}$ & $\begin{array}{l}12.3\left(\mathrm{CH}_{3}\right), 16.8 \\
\left(\mathrm{CH}_{3}\right), 22.1\left(\mathrm{CH}_{2}\right), \\
23.5\left(\mathrm{CH}_{2}\right), 29.4 \\
\left(\mathrm{CH}_{2}\right), 31.8\left(\mathrm{CH}_{2}\right), \\
58.5\left(\mathrm{NCH}_{2}\right), 59.9 \\
\left(\mathrm{OCH}_{2}\right), 126.1 \\
(\mathrm{CH}), 138.1(\mathrm{C}), \\
139.9(\mathrm{CH}), 142.3 \\
(\mathrm{CH}), 144.0(\mathrm{CH}), \\
171.7(\mathrm{CO})\end{array}$ & $\begin{array}{l}- \\
- \\
-\end{array}$ & $\begin{array}{c}1731,1559 \\
1449,1167 \\
1030\end{array}$ & $\begin{array}{c}\left(\mathrm{M}-\mathrm{N}(\mathrm{CN})_{2}\right) \\
236.20 \\
\text { found for } \\
\mathrm{C}_{14} \mathrm{H}_{22} \mathrm{NO}_{2}{ }^{+}\end{array}$ \\
\hline 17 & $\begin{array}{l}1.14\left(\mathrm{t}, 3 \mathrm{H}, \mathrm{J} 7.6, \mathrm{OCH}_{2} \mathrm{CH}_{3}\right), 1.39 \\
\text { (quint, } 2 \mathrm{H}, J 8, \mathrm{CH}_{2} \mathrm{CH}_{2} \mathrm{CH}_{2} \text { ), } \\
\left.1.62 \text { (quint, } 2 \mathrm{H}, \mathrm{J} 8, \mathrm{CH}_{2} \mathrm{CH}_{2} \mathrm{CO}\right), \\
\left.2.03 \text { (quint, } 2 \mathrm{H}, J 8, \mathrm{CH}_{2} \mathrm{CH}_{2} \mathrm{~N}\right), \\
2.26\left(\mathrm{t}, 2 \mathrm{H}, J 8, \mathrm{CH}_{2} \mathrm{CO}\right), 2.60(\mathrm{~s}, \\
\left.3 \mathrm{H}, \mathrm{CH}_{3}\right), 4.01(\mathrm{q}, 2 \mathrm{H}, J 6.8 \\
\left.\mathrm{OCH}_{2} \mathrm{CH}_{3}\right), 4.72(\mathrm{t}, 2 \mathrm{H}, J 8, \\
\left.\mathrm{NCH}_{2} \mathrm{CH}_{2}\right), 8.00(\mathrm{t}, 1 \mathrm{H}, \mathrm{Ar}-\mathrm{H}), \\
8.312(\mathrm{~d}, 1 \mathrm{H}, \mathrm{Ar}-\mathrm{H}), 8.88(\mathrm{~d}, 1 \mathrm{H}, \\
\mathrm{Ar}-\mathrm{H}), 8.93(\mathrm{~s}, 1 \mathrm{H}, \mathrm{Ar}-\mathrm{H})\end{array}$ & $\begin{array}{l}12.2\left(\mathrm{CH}_{3}\right), 16.7 \\
\left(\mathrm{CH}_{3}\right), 22.0\left(\mathrm{CH}_{2}\right), \\
23.3\left(\mathrm{CH}_{2}\right), 29.3 \\
\left(\mathrm{CH}_{2}\right), 31.7\left(\mathrm{CH}_{2}\right), \\
58.3\left(\mathrm{NCH}_{2}\right), 59.5 \\
\left(\mathrm{OCH}_{2}\right), 125.9 \\
(\mathrm{CH}), 138.1(\mathrm{C}), \\
139.9(\mathrm{CH}), 142.3 \\
(\mathrm{CH}), 144.1(\mathrm{CH}), \\
171.3(\mathrm{CO})\end{array}$ & $\begin{array}{l}- \\
- \\
-\end{array}$ & $\begin{array}{l}3132,1731 \\
1556,1447 \\
1165,1032\end{array}$ & $\begin{array}{c}\left(\mathrm{M}-\mathrm{NO}_{3}\right) \\
236.24 \\
\text { found for } \\
\mathrm{C}_{14} \mathrm{H}_{22} \mathrm{NO}_{2}^{+}\end{array}$ \\
\hline 18 & $\begin{array}{l}1.75 \text { (quint, } 2 \mathrm{H}, J \text { 8, } \mathrm{NCH}_{2} \\
\left.\mathrm{CH}_{2} \mathrm{CH}_{2}\right), 2.08 \text { (quint, } 2 \mathrm{H}, J 8, \\
\left.\mathrm{CH}_{2} \mathrm{CH}_{2} \mathrm{CH}_{2} \mathrm{O}\right), 2.48(\mathrm{~s}, 3 \mathrm{H}, \\
\left.\mathrm{CH}_{3}\right), 3.88\left(\mathrm{t}, 2 \mathrm{H}, J 8, \mathrm{NCH}_{2} \mathrm{CH}_{2}\right) \\
4.53\left(\mathrm{t}, 2 \mathrm{H}, J 8, \mathrm{OCH} \mathrm{CH}_{2}\right) \\
\text { 6.76-6.85 (m, 3H, Ar- } \mathrm{H}), 7.14-7.18 \\
(\mathrm{~m}, 2 \mathrm{H}, \mathrm{Ar}-\mathrm{H}), 7.69(\mathrm{~d}, 2 \mathrm{H}, \mathrm{Ar}-\mathrm{H}) \text {, } \\
8.60(\mathrm{~d}, 2 \mathrm{H}, \mathrm{Ar}-\mathrm{H})\end{array}$ & $\begin{array}{l}20.2\left(\mathrm{CH}_{3}\right), 23.9 \\
\left(\mathrm{CH}_{2}\right), 26.6\left(\mathrm{CH}_{2}\right), \\
59.2\left(\mathrm{NCH}_{2}\right), 65.2 \\
(\mathrm{OCH}), 112.8 \\
(\mathrm{CH}), 119.2(\mathrm{CH}), \\
127.3(\mathrm{CH}), 127.9 \\
(\mathrm{CH}), 144.7(\mathrm{CH}), \\
156.9(\mathrm{C}), 157.8(\mathrm{C})\end{array}$ & $\begin{array}{c}-150.84(\mathrm{~s}) \\
-0.99(\mathrm{~s}) \\
-\end{array}$ & $\begin{array}{c}3129,1600 \\
1471,1163 \\
1084,816\end{array}$ & $\begin{array}{c}\left(\mathrm{M}_{\left.-\mathrm{BF}_{4}\right)}\right. \\
242.21 \\
\text { found for } \\
\mathrm{C}_{16} \mathrm{H}_{20} \mathrm{NO}^{+}\end{array}$ \\
\hline 19 & $\begin{array}{l}1.68 \text { (quint, } 2 \mathrm{H}, J \mathrm{~J}, \mathrm{NCH}_{2} \\
\left.\mathrm{CH}_{2} \mathrm{CH}_{2}\right), 2.08 \text { (quint, } 2 \mathrm{H}, J \text {, } \\
\left.\mathrm{CH}_{2} \mathrm{CH}_{2} \mathrm{CH}_{2} \mathrm{O}\right), 2.61(\mathrm{~s}, 3 \mathrm{H}, \\
\left.\mathrm{CH}_{3}\right), 4.00\left(\mathrm{t}, 2 \mathrm{H}, J 8, \mathrm{NCH}_{2} \mathrm{CH}_{2}\right), \\
4.62\left(\mathrm{t}, 2 \mathrm{H}, \mathrm{J} 8, \mathrm{OCH} \mathrm{CH}_{2},\right. \\
6.89-6.93(\mathrm{~m}, 3 \mathrm{H}, \mathrm{Ar}-\mathrm{H}), 7.25-7.29 \\
(\mathrm{~m}, 2 \mathrm{H}, \mathrm{Ar}-\mathrm{H}), 7.94(\mathrm{~d}, 2 \mathrm{H}, \\
\mathrm{Ar}-\mathrm{H}), 8.94(\mathrm{~d}, 2 \mathrm{H}, \mathrm{Ar}-\mathrm{H})\end{array}$ & $\begin{array}{l}20.2\left(\mathrm{CH}_{3}\right), 24.2 \\
\left(\mathrm{CH}_{2}\right), 26.6\left(\mathrm{CH}_{2}\right), \\
58.7\left(\mathrm{NCH}_{2}\right), 65.4 \\
\left(\mathrm{OCH}_{2}\right), 113.2 \\
(\mathrm{CH}), 119.4(\mathrm{CH}), \\
127.3(\mathrm{CH}), 128.3 \\
(\mathrm{CH}), 142.5(\mathrm{CH}), \\
157.3(\mathrm{C}), 157.8(\mathrm{C})\end{array}$ & $\begin{array}{c}-69.43 \\
-71.32(\mathrm{~d}) \\
- \\
-131.01 \\
-157.36 \\
\text { (sept) }\end{array}$ & $\begin{array}{c}3135,1601 \\
1470,1166, \\
1083,815\end{array}$ & $\begin{array}{c}\left(\mathrm{M}-\mathrm{PF}_{6}\right) \\
242.23 \\
\text { found for } \\
\mathrm{C}_{16} \mathrm{H}_{20} \mathrm{NO}^{+}\end{array}$ \\
\hline 20 & $\begin{array}{l}1.68 \text { (quint, } 2 \mathrm{H}, \mathrm{J} 8, \mathrm{NCH}_{2} \\
\left.\mathrm{CH}_{2} \mathrm{CH}_{2}\right), 2.03 \text { (quint, } 2 \mathrm{H}, J \text {, } \\
\left.\mathrm{CH}_{2} \mathrm{CH}_{2} \mathrm{CH}_{2} \mathrm{O}\right), 2.40(\mathrm{~s}, 3 \mathrm{H}, \\
\left.\mathrm{CH}_{3}\right), 3.79\left(\mathrm{t}, 2 \mathrm{H}, \mathrm{J} 8, \mathrm{NCH}_{2} \mathrm{CH}_{2}\right), \\
4.71\left(\mathrm{t}, 2 \mathrm{H}, \mathrm{J} 8, \mathrm{OCH} \mathrm{CH}_{2}\right) \\
\text { 6.64-6.73 (m, 3H, Ar- } \mathrm{H}), \\
\text { 7.02-7.07 (m, 2H, Ar- } \mathrm{H}), 7.65(\mathrm{~d}, \\
2 \mathrm{H}, \mathrm{Ar}-\mathrm{H}), 9.09(\mathrm{~d}, 2 \mathrm{H}, \mathrm{Ar}-\mathrm{H})\end{array}$ & $\begin{array}{l}20.1\left(\mathrm{CH}_{3}\right), 23.7 \\
\left(\mathrm{CH}_{2}\right), 26.7\left(\mathrm{CH}_{2}\right), \\
58.5\left(\mathrm{NCH}_{2}\right), 64.8 \\
(\mathrm{OCH}), 112.5 \\
(\mathrm{CH}), 114.9(\mathrm{CH}), \\
118.9(\mathrm{CH}), 126.9 \\
(\mathrm{CH}), 127.6(\mathrm{CH}), \\
142.2(\mathrm{CH}), 156.6 \\
(\mathrm{C}), 157.1(\mathrm{C})\end{array}$ & $\begin{array}{c}-75.19(\mathrm{~s}) \\
- \\
-\end{array}$ & $\begin{array}{c}3130,1599 \\
1469,1167 \\
1083,815\end{array}$ & $\begin{array}{c}\left(\mathrm{M}-\mathrm{CF}_{3} \mathrm{CO}_{2}\right) \\
242.23 \\
\text { found for } \\
\mathrm{C}_{16} \mathrm{H}_{20} \mathrm{NO}^{+}\end{array}$ \\
\hline
\end{tabular}


Table IV. continued

\begin{tabular}{|c|c|c|c|c|c|}
\hline 21 & $\begin{array}{l}1.82 \text { (quint, } 2 \mathrm{H}, \mathrm{J} 8, \mathrm{NCH}_{2} \\
\left.\mathrm{CH}_{2} \mathrm{CH}_{2}\right), 2.18 \text { (quint, } 2 \mathrm{H}, \mathrm{J} 8, \\
\left.\mathrm{CH}_{2} \mathrm{CH}_{2} \mathrm{CH}_{2} \mathrm{O}\right), 2.85(\mathrm{~s}, 3 \mathrm{H}, \\
\left.\mathrm{CH}_{3}\right), 3.93\left(\mathrm{t}, 2 \mathrm{H}, \mathrm{J} 8, \mathrm{NCH}_{2} \mathrm{CH}_{2}\right), \\
4.73\left(\mathrm{t}, 2 \mathrm{H}, \mathrm{J} 8, \mathrm{OCH} \mathrm{CH}_{2},\right. \\
6.78-6.85(\mathrm{~m}, 3 \mathrm{H}, \mathrm{Ar}-\mathrm{H}), 7.15-7.19 \\
(\mathrm{~m}, 2 \mathrm{H}, \mathrm{Ar}-\mathrm{H}), 7.80(\mathrm{~d}, 2 \mathrm{H}, \\
\mathrm{Ar}-\mathrm{H}), 8.82(\mathrm{~d}, 2 \mathrm{H}, \mathrm{Ar}-\mathrm{H})\end{array}$ & $\begin{array}{l}20.4\left(\mathrm{CH}_{3}\right), 23.9 \\
\left(\mathrm{CH}_{2}\right), 26.7\left(\mathrm{CH}_{2}\right), \\
59.2\left(\mathrm{NCH}_{2}\right), 65.0 \\
\left(\mathrm{OCH}_{2}\right), 112.6 \\
(\mathrm{CH}), 118.9(\mathrm{CH}), \\
127.2(\mathrm{CH}), 127.6 \\
(\mathrm{CH}), 141.6(\mathrm{CH}), \\
156.6(\mathrm{C}), 157.6(\mathrm{C})\end{array}$ & $\begin{array}{l}- \\
- \\
-\end{array}$ & $\begin{array}{c}3133,1600 \\
1472,1165 \\
1083,814\end{array}$ & $\begin{array}{c}\text { (M- NCS) } \\
242.20 \\
\text { found for } \\
\mathrm{C}_{16} \mathrm{H}_{20} \mathrm{NO}^{+}\end{array}$ \\
\hline 22 & $\begin{array}{l}1.81 \text { (quint, } 2 \mathrm{H}, J 8, \mathrm{NCH}_{2} \\
\left.\mathrm{CH}_{2} \mathrm{CH}_{2}\right), 2.16 \text { (quint, } 2 \mathrm{H}, J \text {, } \\
\left.\mathrm{CH}_{2} \mathrm{CH}_{2} \mathrm{CH}_{2} \mathrm{O}\right), 2.57(\mathrm{~s}, 3 \mathrm{H}, \\
\left.\mathrm{CH}_{3}\right), 3.93\left(\mathrm{t}, 2 \mathrm{H}, \mathrm{J} 8, \mathrm{NCH}_{2} \mathrm{CH}_{2}\right), \\
4.71\left(\mathrm{t}, 2 \mathrm{H}, \mathrm{J} 8, \mathrm{OCH} \mathrm{CH}_{2}\right), \\
6.78-6.87(\mathrm{~m}, 3 \mathrm{H}, \mathrm{Ar}-\mathrm{H}), \\
7.16-7.20(\mathrm{~m}, 2 \mathrm{H}, \mathrm{Ar}-\mathrm{H}), 7.75(\mathrm{~d}, \\
\text { 2H, Ar- } \mathrm{H}), 8.91(\mathrm{~d}, 2 \mathrm{H}, \mathrm{Ar}-\mathrm{H})\end{array}$ & $\begin{array}{l}20.3\left(\mathrm{CH}_{3}\right), 23.7 \\
\left(\mathrm{CH}_{2}\right), 26.8\left(\mathrm{CH}_{2}\right), \\
58.9\left(\mathrm{NCH}_{2}\right), 64.8 \\
\left(\mathrm{OCH}_{2}\right), 112.5 \\
(\mathrm{CH}), 119.0(\mathrm{CH}), \\
127.1(\mathrm{CH}), 127.6 \\
(\mathrm{CH}), 141.7(\mathrm{CH}), \\
156.5(\mathrm{C}), 157.6(\mathrm{C})\end{array}$ & $\begin{array}{l}- \\
- \\
-\end{array}$ & $\begin{array}{c}3131,1599 \\
1471,1165 \\
1082,814\end{array}$ & $\begin{array}{c}\left(\mathrm{M}-\mathrm{N}(\mathrm{CN})_{2}\right) \\
242.21 \\
\text { found for } \\
\mathrm{C}_{16} \mathrm{H}_{20} \mathrm{NO}^{+}\end{array}$ \\
\hline 23 & $\begin{array}{l}1.70 \text { (quint, } 2 \mathrm{H}, \mathrm{J} 8, \mathrm{NCH}_{2} \\
\left.\mathrm{CH}_{2} \mathrm{CH}_{2}\right), 2.06(\text { quint, } 2 \mathrm{H}, \mathrm{J} 8, \\
\left.\mathrm{CH}_{2} \mathrm{CH}_{2} \mathrm{CH}_{2} \mathrm{O}\right), 2.61(\mathrm{~s}, 3 \mathrm{H}, \\
\left.\mathrm{CH}_{3}\right), 3.79\left(\mathrm{t}, 2 \mathrm{H}, \mathrm{J} 8, \mathrm{NCH}_{2} \mathrm{CH}_{2}\right), \\
4.78\left(\mathrm{t}, 2 \mathrm{H}, \mathrm{J} 8, \mathrm{OCH} \mathrm{CH}_{2}\right), \\
6.63-6.73(\mathrm{~m}, 3 \mathrm{H}, \mathrm{Ar}-\mathrm{H}), \\
7.02-7.06(\mathrm{~m}, 2 \mathrm{H}, \mathrm{Ar}-\mathrm{H}), 7.66(\mathrm{~d}, \\
2 \mathrm{H}, \mathrm{Ar}-\mathrm{H}), 9.22(\mathrm{~d}, 2 \mathrm{H}, \mathrm{Ar}-\mathrm{H})\end{array}$ & $\begin{array}{l}22.1\left(\mathrm{CH}_{3}\right), 25.6 \\
\left(\mathrm{CH}_{2}\right), 28.7\left(\mathrm{CH}_{2}\right), \\
60.4\left(\mathrm{NCH}_{2}\right), 66.8 \\
\left(\mathrm{OCH}_{2}\right), 120.8 \\
(\mathrm{CH}), 128.8(\mathrm{CH}), \\
129.4(\mathrm{CH}), 144.2 \\
(\mathrm{CH}), 158.5(\mathrm{C}), \\
158.9(\mathrm{C})\end{array}$ & $\begin{array}{l}- \\
- \\
-\end{array}$ & $\begin{array}{c}3130,1598 \\
1470,1167 \\
1084,813\end{array}$ & $\begin{array}{c}\left(\mathrm{M}-\mathrm{NO}_{3}\right) \\
242.23 \\
\text { found for } \\
\mathrm{C}_{16} \mathrm{H}_{20} \mathrm{NO}^{+}\end{array}$ \\
\hline 24 & $\begin{array}{l}1.10\left(\mathrm{t}, 3 \mathrm{H}, J 7.6, \mathrm{OCH}_{2} \mathrm{CH}_{3}\right), 1.23 \\
\text { (quint, } 2 \mathrm{H}, J 8, \mathrm{CH}_{2} \mathrm{CH}_{2} \mathrm{CH}_{2} \text { ), } \\
\left.1.50 \text { (quint, } 2 \mathrm{H}, J 8, \mathrm{CH}_{2} \mathrm{CH}_{2} \mathrm{CO}\right), \\
\left.1.89 \text { (quint, } 2 \mathrm{H}, J 8, \mathrm{CH}_{2} \mathrm{CH}_{2} \mathrm{~N}\right) \text {, } \\
2.23\left(\mathrm{t}, 2 \mathrm{H}, J 8, \mathrm{CH}_{2} \mathrm{CO}\right), 2.59(\mathrm{~s}, \\
\left.3 \mathrm{H}, \mathrm{CH}_{3}\right), 3.98(\mathrm{q}, 2 \mathrm{H}, J 6.8, \\
\left.\mathrm{OCH}_{2} \mathrm{CH}_{3}\right), 4.61(\mathrm{t}, 2 \mathrm{H}, J 8, \\
\left.\mathrm{NCH}_{2} \mathrm{CH}_{2}\right), 8.02(\mathrm{~d}, 2 \mathrm{H}, \mathrm{Ar}-\mathrm{H}), \\
9.08(\mathrm{~d}, 2 \mathrm{H}, \mathrm{Ar}-\mathrm{H})\end{array}$ & $\begin{array}{l}14.0\left(\mathrm{CH}_{3}\right), 21.3 \\
\left(\mathrm{CH}_{3}\right), 23.7\left(\mathrm{CH}_{2}\right), \\
24.6\left(\mathrm{CH}_{2}\right), 20.2 \\
\left(\mathrm{CH}_{2}\right), 33.1\left(\mathrm{CH}_{2}\right), \\
59.5\left(\mathrm{NCH}_{2}\right), 59.7 \\
\left(\mathrm{OCH}_{2}\right), 128.2 \\
(\mathrm{CH}), 143.7(\mathrm{CH}), \\
158.7(\mathrm{C}), 171.7 \\
(\mathrm{CO})\end{array}$ & $\begin{array}{c}-150.84 \\
-0.99 \\
- \\
-\end{array}$ & $\begin{array}{l}3132,1731 \\
1556,1447 \\
1165,1032\end{array}$ & $\begin{array}{c}\left(\mathrm{M}_{-} \mathrm{BF}_{4}\right) \\
236.20 \\
\text { found for } \\
\mathrm{C}_{14} \mathrm{H}_{22} \mathrm{NO}_{2}{ }^{+}\end{array}$ \\
\hline 25 & $\begin{array}{l}1.16\left(\mathrm{t}, 3 \mathrm{H}, J \mathrm{~J} .6, \mathrm{OCH}_{2} \mathrm{CH}_{3}\right), 1.29 \\
\left.\text { (quint, } 2 \mathrm{H}, J 8, \mathrm{CH}_{2} \mathrm{CH}_{2} \mathrm{CH}_{2}\right), \\
\left.1.57 \text { (quint, } 2 \mathrm{H}, J 8, \mathrm{CH}_{2} \mathrm{CH}_{2} \mathrm{CO}\right), \\
1.94\left(\text { quint, } 2 \mathrm{H}, J 8, \mathrm{CH}_{2} \mathrm{CH}_{2} \mathrm{~N}\right), \\
2.29\left(\mathrm{t}, 2 \mathrm{H}, J 8, \mathrm{CH}_{2} \mathrm{CO}\right), 2.61(\mathrm{~s}, \\
\left.3 \mathrm{H}, \mathrm{CH}_{3}\right), 4.04(\mathrm{q}, 2 \mathrm{H}, J 6.8, \\
\left.\mathrm{OCH}_{2} \mathrm{CH}_{3}\right), 4.53(\mathrm{t}, 2 \mathrm{H}, J 8, \\
\left.\mathrm{NCH}_{2} \mathrm{CH}_{2}\right), 7.96(\mathrm{~d}, 2 \mathrm{H}, \mathrm{Ar}-\mathrm{H}), \\
8.90(\mathrm{~d}, 2 \mathrm{H}, \mathrm{Ar}-\mathrm{H})\end{array}$ & $\begin{array}{l}13.9\left(\mathrm{CH}_{3}\right), 21.1 \\
\left(\mathrm{CH}_{3}\right), 23.6\left(\mathrm{CH}_{2}\right), \\
24.6\left(\mathrm{CH}_{2}\right), 30.1 \\
\left(\mathrm{CH}_{2}\right), 33.0\left(\mathrm{CH}_{2}\right), \\
59.6\left(\mathrm{NCH}_{2}\right), 59.7 \\
(\mathrm{OCH}), 128.2 \\
(\mathrm{CH}), 143.5 \\
(\mathrm{CH}), 158.8(\mathrm{C}), \\
172.7(\mathrm{CO})\end{array}$ & $\begin{array}{c}-69.34 \\
-71.23(\mathrm{~d}) \\
- \\
-131.03 \\
-157.38 \\
\text { (sept) }\end{array}$ & $\begin{array}{l}3132,1729 \\
1560,1449 \\
1168,1034\end{array}$ & $\begin{array}{c}\left(\mathrm{M}-\mathrm{PF}_{6}\right) \\
236.23 \\
\text { found for } \\
\mathrm{C}_{14} \mathrm{H}_{22} \mathrm{NO}_{2}^{+}\end{array}$ \\
\hline
\end{tabular}


Table IV. continued

\begin{tabular}{|c|c|c|c|c|c|}
\hline 26 & $\begin{array}{l}1.13\left(\mathrm{t}, 3 \mathrm{H}, J \mathrm{~J} .6, \mathrm{OCH}_{2} \mathrm{CH}_{3}\right), 1.23 \\
\text { (quint, } 2 \mathrm{H}, J 8, \mathrm{CH}_{2} \mathrm{CH}_{2} \mathrm{CH}_{2} \text { ), } \\
\left.1.53 \text { (quint, } 2 \mathrm{H}, J 8, \mathrm{CH}_{2} \mathrm{CH}_{2} \mathrm{CO}\right), \\
\left.1.89 \text { (quint, } 2 \mathrm{H}, J 8, \mathrm{CH}_{2} \mathrm{CH}_{2} \mathrm{~N}\right), \\
2.26\left(\mathrm{t}, 2 \mathrm{H}, J 8, \mathrm{CH}_{2} \mathrm{CO}\right), 2.59(\mathrm{~s}, \\
\left.3 \mathrm{H}, \mathrm{CH}_{3}\right), 4.02(\mathrm{q}, 2 \mathrm{H}, J 6.8, \\
\left.\mathrm{OCH}_{2} \mathrm{CH}_{3}\right), 4.58(\mathrm{t}, 2 \mathrm{H}, J 8, \\
\left.\mathrm{NCH}_{2} \mathrm{CH}_{2}\right), 8.01(\mathrm{~d}, 2 \mathrm{H}, \mathrm{Ar}-\mathrm{H}), \\
9.05(\mathrm{~d}, 2 \mathrm{H}, \mathrm{Ar}-\mathrm{H})\end{array}$ & $\begin{array}{l}12.9\left(\mathrm{CH}_{3}\right), 20.1 \\
\left(\mathrm{CH}_{3}\right), 22.6\left(\mathrm{CH}_{2}\right), \\
23.6\left(\mathrm{CH}_{2}\right), 29.2 \\
\left(\mathrm{CH}_{2}\right), 32.0\left(\mathrm{CH}_{2}\right), \\
58.5\left(\mathrm{NCH}_{2}\right), 58.6 \\
\left(\mathrm{OCH}_{2}\right), 127.2 \\
(\mathrm{CH}), 142.7(\mathrm{CH}), \\
157.6(\mathrm{C}), 171.6 \\
(\mathrm{CO})\end{array}$ & $\begin{array}{c}-73.76(\mathrm{~s}) \\
- \\
-\end{array}$ & $\begin{array}{c}3133,1731, \\
1559,1449, \\
1167,1030\end{array}$ & $\begin{array}{c}\left(\mathrm{M}-\mathrm{CF}_{3} \mathrm{CO}_{2}\right) \\
236.22 \\
\text { found for } \\
\mathrm{C}_{14} \mathrm{H}_{22} \mathrm{NO}_{2}^{+}\end{array}$ \\
\hline 27 & $\begin{array}{l}\left.1.13 \text { (t, 3H, } J \text { 7.6, } \mathrm{OCH}_{2} \mathrm{CH}_{3}\right), 1.26 \\
\text { (quint, } 2 \mathrm{H}, J 8, \mathrm{CH}_{2} \mathrm{CH}_{2} \mathrm{CH}_{2} \text { ), } \\
1.53 \text { (quint, } 2 \mathrm{H}, J 8, \mathrm{CH}_{2} \mathrm{CH}_{2} \mathrm{CO} \text { ), } \\
\left.1.89 \text { (quint, } 2 \mathrm{H}, \mathrm{J} 8, \mathrm{CH}_{2} \mathrm{CH}_{2} \mathrm{~N}\right), \\
2.26\left(\mathrm{t}, 2 \mathrm{H}, J 8, \mathrm{CH}_{2} \mathrm{CO}\right), 2.61(\mathrm{~s}, \\
\left.3 \mathrm{H}, \mathrm{CH}_{3}\right), 4.01(\mathrm{q}, 2 \mathrm{H}, J 6.8, \\
\left.\mathrm{OCH}_{2} \mathrm{CH}_{3}\right), 4.55(\mathrm{t}, 2 \mathrm{H}, J 8, \\
\left.\mathrm{NCH}_{2} \mathrm{CH}_{2}\right), 8.00(\mathrm{~d}, 2 \mathrm{H}, \mathrm{Ar}-\mathrm{H}), \\
9.94(\mathrm{~d}, 2 \mathrm{H}, \mathrm{Ar}-\mathrm{H})\end{array}$ & $\begin{array}{l}12.9\left(\mathrm{CH}_{3}\right), 20.2 \\
\left(\mathrm{CH}_{3}\right), 22.5\left(\mathrm{CH}_{2}\right), \\
23.6\left(\mathrm{CH}_{2}\right), 29.0 \\
\left(\mathrm{CH}_{2}\right), 31.9\left(\mathrm{CH}_{2}\right), \\
58.5\left(\mathrm{NCH}_{2}\right), 58.6 \\
\left(\mathrm{OCH}_{2}\right), 127.2 \\
(\mathrm{CH}), 142.4(\mathrm{CH}), \\
157.6(\mathrm{C}), 171.5 \\
(\mathrm{CO})\end{array}$ & $\begin{array}{l}- \\
- \\
-\end{array}$ & $\begin{array}{l}3133,1729, \\
1559,1450, \\
1166,1030\end{array}$ & $\begin{array}{c}\text { (M-NCS) } \\
236.23 \\
\text { found for } \\
\mathrm{C}_{14} \mathrm{H}_{22} \mathrm{NO}_{2}^{+}\end{array}$ \\
\hline 28 & $\begin{array}{l}\left.1.14 \text { (t, 3H, J 7.6, } \mathrm{OCH}_{2} \mathrm{CH}_{3}\right), 1.26 \\
\text { (quint, } 2 \mathrm{H}, J 8, \mathrm{CH}_{2} \mathrm{CH}_{2} \mathrm{CH}_{2} \text { ), } \\
1.52 \text { (quint, } 2 \mathrm{H}, J 8, \mathrm{CH}_{2} \mathrm{CH}_{2} \mathrm{CO} \text { ), } \\
1.90 \text { (quint, } 2 \mathrm{H}, \mathrm{J} 8, \mathrm{CH}_{2} \mathrm{CH}_{2} \mathrm{~N} \text { ), } \\
2.27\left(\mathrm{t}, 2 \mathrm{H}, J 8, \mathrm{CH}_{2} \mathrm{CO}\right), 2.60(\mathrm{~s}, \\
\left.3 \mathrm{H}, \mathrm{CH}_{3}\right), 4.02(\mathrm{q}, 2 \mathrm{H}, \mathrm{J} 6.8 \\
\left.\mathrm{OCH}_{2} \mathrm{CH}_{3}\right), 4.54(\mathrm{t}, 2 \mathrm{H}, J 8, \\
\left.\mathrm{NCH}_{2} \mathrm{CH}_{2}\right), 7.99(\mathrm{~d}, 2 \mathrm{H}, \mathrm{Ar}-\mathrm{H}), \\
8.96(\mathrm{~d}, 2 \mathrm{H}, \mathrm{Ar}-\mathrm{H})\end{array}$ & $\begin{array}{l}14.0\left(\mathrm{CH}_{3}\right), 21.2 \\
\left(\mathrm{CH}_{3}\right), 23.6\left(\mathrm{CH}_{2}\right), \\
24.7\left(\mathrm{CH}_{2}\right), 30.2 \\
\left(\mathrm{CH}_{2}\right), 33.1\left(\mathrm{CH}_{2}\right), \\
59.6\left(\mathrm{NCH}_{2}\right), 59.7 \\
\left(\mathrm{OCH}_{2}\right), 128.3 \\
(\mathrm{CH}), 143.6(\mathrm{CH}), \\
158.7(\mathrm{C}), 172.7 \\
(\mathrm{CO})\end{array}$ & $\begin{array}{l}- \\
- \\
-\end{array}$ & $\begin{array}{c}3132,1730 \\
1558-1448, \\
1167,1032\end{array}$ & $\begin{array}{c}\left(\mathrm{M}-\mathrm{N}(\mathrm{CN})_{2}\right) \\
236.20 \\
\text { found for } \\
\mathrm{C}_{14} \mathrm{H}_{22} \mathrm{NO}_{2}^{+}\end{array}$ \\
\hline 29 & 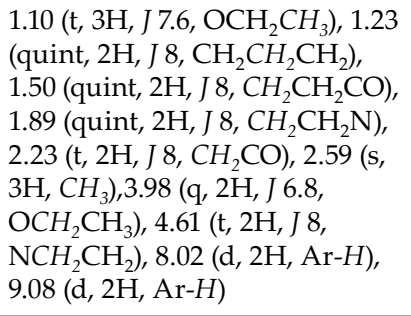 & $\begin{array}{l}14.0\left(\mathrm{CH}_{3}\right), 21.3 \\
\left(\mathrm{CH}_{3}\right), 23.7\left(\mathrm{CH}_{2}\right), \\
24.6\left(\mathrm{CH}_{2}\right), 20.2 \\
\left(\mathrm{CH}_{2}\right), 33.1\left(\mathrm{CH}_{2}\right), \\
59.5\left(\mathrm{NCH}_{2}\right), 59.7 \\
\left(\mathrm{OCH}_{2}\right), 128.2 \\
(\mathrm{CH}), 143.7(\mathrm{CH}), \\
158.7(\mathrm{C}), 171.7 \\
(\mathrm{CO})\end{array}$ & $\begin{array}{l}- \\
- \\
-\end{array}$ & $\begin{array}{c}3131,1728, \\
1559,1449, \\
1167,1032\end{array}$ & $\begin{array}{c}\left(\mathrm{M}-\mathrm{NO}_{3}\right) \\
236.20 \\
\text { found for } \\
\mathrm{C}_{14} \mathrm{H}_{22} \mathrm{NO}_{2}^{+}\end{array}$ \\
\hline 30 & $\begin{array}{l}\delta 1.27 \text { (quint, } 2 \mathrm{H}, J \text { 8, } \\
\mathrm{CH}_{2} \mathrm{CH}_{2} \mathrm{CH}_{2} \text { ), } 1.52 \text { (quint, } 2 \mathrm{H}, J \\
\left.8, \mathrm{CH}_{2} \mathrm{CH}_{2} \mathrm{CO}\right), 1.90 \text { (quint, } 2 \mathrm{H}, \\
\left.J 8, \mathrm{CH}_{2} \mathrm{CH}_{2} \mathrm{~N}\right), 2.20(\mathrm{t}, 2 \mathrm{H}, J 8, \\
\left.\mathrm{CH}_{2} \mathrm{CO}\right), 2.59\left(\mathrm{~s}, 3 \mathrm{H}, \mathrm{CH}_{3}\right), 4.52 \\
\left(\mathrm{t}, 2 \mathrm{H}, \mathrm{J} 6.8, \mathrm{NCH}_{2} \mathrm{CH}_{2}\right), 7.95(\mathrm{~d}, \\
2 \mathrm{H}, \mathrm{Ar}-\mathrm{H}), 8.89(\mathrm{~d}, 2 \mathrm{H}, \mathrm{Ar}-\mathrm{H}), \\
12.03\left(\mathrm{~s}, 1 \mathrm{H}, \mathrm{CO}_{2} \mathrm{H}\right)\end{array}$ & $\begin{array}{l}20.2\left(\mathrm{CH}_{3}\right), 22.7 \\
\left(\mathrm{CH}_{2}\right), 23.8\left(\mathrm{CH}_{2}\right), \\
29.2\left(\mathrm{CH}_{2}\right), 32.2 \\
\left(\mathrm{CH}_{2}\right), 58.7 \\
\left(\mathrm{NCH}_{2}\right), 127.3 \\
(\mathrm{CH}), 142.5(\mathrm{CH}) \text {, } \\
157.8(\mathrm{C}), 173.4 \\
(\mathrm{CO})\end{array}$ & $\begin{array}{c}-148.23(\mathrm{~s}) \\
-1.23(\mathrm{~s}) \\
-\end{array}$ & $\begin{array}{c}3252,3130, \\
1730,1560, \\
1448,1166, \\
1031\end{array}$ & $\begin{array}{c}\left(\mathrm{M}-\mathrm{BF}_{4}\right) \\
208.23 \\
\text { found for } \\
\mathrm{C}_{12} \mathrm{H}_{18} \mathrm{NO}_{2}^{+}\end{array}$ \\
\hline
\end{tabular}


Table IV. continued

\begin{tabular}{|c|c|c|c|c|c|}
\hline 31 & $\begin{array}{l}\delta 1.26 \text { (quint, 2H, J 8, } \\
\mathrm{CH}_{2} \mathrm{CH}_{2} \mathrm{CH}_{2} \text { ), } 1.54 \text { (quint, } 2 \mathrm{H}, \\
\left.J \text { 8, } \mathrm{CH}_{2} \mathrm{CH}_{2} \mathrm{CO}\right), 1.89 \text { (quint, } \\
\left.2 \mathrm{H}, J 8, \mathrm{CH}_{2} \mathrm{CH}_{2} \mathrm{~N}\right), 2.22(\mathrm{t}, 2 \mathrm{H}, \\
\left.J \text { 8, } \mathrm{CH}_{2} \mathrm{CO}\right), 2.60\left(\mathrm{~s}, 3 \mathrm{H}, \mathrm{CH}_{3}\right), \\
4.52\left(\mathrm{t}, 2 \mathrm{H}, \mathrm{J} 6.8, \mathrm{NCH}_{2} \mathrm{CH}_{2}\right), \\
7.96(\mathrm{~d}, 2 \mathrm{H}, \mathrm{Ar}-\mathrm{H}), 8.90(\mathrm{~d}, 2 \mathrm{H}, \\
\text { Ar- } \mathrm{H}), 12.00\left(\mathrm{~s}, 1 \mathrm{H}, \mathrm{CO}_{2} \mathrm{H}\right)\end{array}$ & $\begin{array}{l}20.2\left(\mathrm{CH}_{3}\right), 22.7 \\
\left(\mathrm{CH}_{2}\right), 23.8\left(\mathrm{CH}_{2}\right), \\
29.2\left(\mathrm{CH}_{2}\right), 32.2 \\
\left(\mathrm{CH}_{2}\right), 58.8 \\
\left(\mathrm{NCH}_{2}\right), 127.3 \\
(\mathrm{CH}), 142.6(\mathrm{CH}), \\
157.8(\mathrm{C}), 173.3 \\
(\mathrm{CO})\end{array}$ & $\begin{array}{c}-69.25 \\
-71.14 \\
- \\
-131.04 \\
-157.39 \\
\text { (sept) }\end{array}$ & $\begin{array}{c}3259,3132, \\
1731,1558, \\
1446,1168, \\
1029\end{array}$ & $\begin{array}{c}\left(\mathrm{M}-\mathrm{PF}_{6}\right) \\
208.23 \\
\text { found for } \\
\mathrm{C}_{12} \mathrm{H}_{18} \mathrm{NO}_{2}^{+}\end{array}$ \\
\hline 32 & $\begin{array}{l}\delta 1.28 \text { (quint, } 2 \mathrm{H}, J \text {, } \\
\mathrm{CH}_{2} \mathrm{CH}_{2} \mathrm{CH}_{2} \text { ), } 1.54 \text { (quint, } 2 \mathrm{H}, \\
\left.J 8, \mathrm{CH}_{2} \mathrm{CH}_{2} \mathrm{CO}\right), 1.89 \text { (quint, } \\
\left.2 \mathrm{H}, J 8, \mathrm{CH}_{2} \mathrm{CH}_{2} \mathrm{~N}\right), 2.22(\mathrm{t}, 2 \mathrm{H}, \\
\left.J \text { 8, } \mathrm{CH}_{2} \mathrm{CO}\right), 2.63\left(\mathrm{~s}, 3 \mathrm{H}, \mathrm{CH}_{3}\right), \\
4.59\left(\mathrm{t}, 2 \mathrm{H}, \mathrm{J} 6.8, \mathrm{NCH}_{2} \mathrm{CH}_{2}\right), \\
8.02(\mathrm{~d}, 2 \mathrm{H}, \mathrm{Ar}-\mathrm{H}), 9.04(\mathrm{~d}, 2 \mathrm{H}, \\
\text { Ar- } \mathrm{H})\end{array}$ & $\begin{array}{l}20.1\left(\mathrm{CH}_{3}\right), 22.6 \\
\left(\mathrm{CH}_{2}\right), 23.7\left(\mathrm{CH}_{2}\right), \\
29.2\left(\mathrm{CH}_{2}\right), 32.3 \\
\left(\mathrm{CH}_{2}\right), 58.5 \\
\left(\mathrm{NCH}_{2}\right), 127.2 \\
(\mathrm{CH}), 142.7(\mathrm{CH}), \\
157.6(\mathrm{C}), 173.2 \\
(\mathrm{CO})\end{array}$ & $\begin{array}{c}-73.73(\mathrm{~s}) \\
- \\
-\end{array}$ & $\begin{array}{c}3253,3132, \\
1729, \\
1561-1450, \\
1166,1030\end{array}$ & $\begin{array}{c}\left(\mathrm{M}-\mathrm{CF}_{3} \mathrm{CO}_{2}\right) \\
208.21 \\
\text { found for } \\
\mathrm{C}_{12} \mathrm{H}_{18} \mathrm{NO}_{2}^{+}\end{array}$ \\
\hline 33 & $\begin{array}{l}\delta 1.27 \text { (quint, 2H, J 8, } \\
\mathrm{CH}_{2} \mathrm{CH}_{2} \mathrm{CH}_{2} \text { ), } 1.53 \text { (quint, } 2 \mathrm{H}, \\
J \text { 8, } \mathrm{CH}_{2} \mathrm{CH}_{2} \mathrm{CO} \text { ), } 1.89 \text { (quint, } \\
2 \mathrm{H}, \mathrm{J} 8, \mathrm{CH}_{2} \mathrm{CH}_{2} \mathrm{~N} \text { ), } 2.22(\mathrm{t}, 2 \mathrm{H}, \\
\left.\mathrm{J} 8, \mathrm{CH}_{2} \mathrm{CO}\right), 2.62\left(\mathrm{~s}, 3 \mathrm{H}, \mathrm{CH}_{3}\right), \\
4.54\left(\mathrm{t}, 2 \mathrm{H}, \mathrm{J} 6.8, \mathrm{NCH}_{2} \mathrm{CH}_{2}\right), \\
8.01(\mathrm{~d}, 2 \mathrm{H}, \mathrm{Ar}-\mathrm{H}), 8.94(\mathrm{~d}, 2 \mathrm{H}, \\
\text { Ar- } \mathrm{H}), 12.04\left(\mathrm{~s}, 1 \mathrm{H}, \mathrm{CO}_{2} \mathrm{H}\right)\end{array}$ & $\begin{array}{l}21.3\left(\mathrm{CH}_{3}\right), 23.7 \\
\left(\mathrm{CH}_{2}\right), 24.8\left(\mathrm{CH}_{2}\right) \\
30.2\left(\mathrm{CH}_{2}\right), 33.3 \\
\left(\mathrm{CH}_{2}\right), 59.7 \\
\left(\mathrm{NCH}_{2}\right), 128.3 \\
(\mathrm{CH}), 143.5(\mathrm{CH}) \\
158.7(\mathrm{C}), 174.2 \\
(\mathrm{CO})\end{array}$ & - & $\begin{array}{c}3255,3132, \\
1728,1563, \\
1451,1165, \\
1031\end{array}$ & $\begin{array}{c}\text { (M-NCS) } \\
208.20 \\
\text { found for } \\
\mathrm{C}_{12} \mathrm{H}_{18} \mathrm{NO}_{2}^{+}\end{array}$ \\
\hline 34 & $\begin{array}{l}\delta 1.25 \text { (quint, 2H, J 8, } \\
\mathrm{CH}_{2} \mathrm{CH}_{2} \mathrm{CH}_{2} \text { ), } 1.51 \text { (quint, 2H, } \\
\left.J \text { 8, } \mathrm{CH}_{2} \mathrm{CH}_{2} \mathrm{CO}\right), 1.89 \text { (quint, } 2 \mathrm{H}, \\
\left.J 8, \mathrm{CH}_{2} \mathrm{CH}_{2} \mathrm{~N}\right), 2.23(\mathrm{t}, 2 \mathrm{H}, J 8, \\
\left.\mathrm{CH}_{2} \mathrm{CO}\right), 2.60\left(\mathrm{~s}, 3 \mathrm{H}, \mathrm{CH}_{3}\right), 4.55 \\
\left(\mathrm{t}, 2 \mathrm{H}, J 6.8, \mathrm{NCH}_{2} \mathrm{CH}_{2}\right), 7.98(\mathrm{~d}, \\
2 \mathrm{H}, \mathrm{Ar}-\mathrm{H}), 8.97(\mathrm{~d}, 2 \mathrm{H}, \mathrm{Ar}-\mathrm{H})\end{array}$ & $\begin{array}{l}20.1\left(\mathrm{CH}_{3}\right), 22.9 \\
\left(\mathrm{CH}_{2}\right), 23.8\left(\mathrm{CH}_{2}\right), \\
29.2\left(\mathrm{CH}_{2}\right), 33.0 \\
\left(\mathrm{CH}_{2}\right), 58.5 \\
\left(\mathrm{NCH}_{2}\right), 127.1 \\
(\mathrm{CH}), 142.5(\mathrm{CH}), \\
157.6(\mathrm{C}), 173.3 \\
(\mathrm{CO})\end{array}$ & - & $\begin{array}{c}3256,3133, \\
1733,1560, \\
1448,1165, \\
1031\end{array}$ & $\begin{array}{c}\left(\mathrm{M}-\mathrm{N}(\mathrm{CN})_{2}\right) \\
208.23 \\
\text { found for } \\
\mathrm{C}_{12} \mathrm{H}_{18} \mathrm{NO}_{2}^{+}\end{array}$ \\
\hline 35 & $\begin{array}{l}\delta 1.27 \text { (quint, 2H, J 8, } \\
\mathrm{CH}_{2} \mathrm{CH}_{2} \mathrm{CH}_{2} \text { ), } 1.54 \text { (quint, } 2 \mathrm{H}, \\
J \text { 8, } \mathrm{CH}_{2} \mathrm{CH}_{2} \mathrm{CO} \text { ), } 1.89 \text { (quint, } \\
\left.2 \mathrm{H}, \mathrm{J} 8, \mathrm{CH}_{2} \mathrm{CH}_{2} \mathrm{~N}\right), 2.23(\mathrm{t}, 2 \mathrm{H}, \\
\left.\mathrm{J} 8, \mathrm{CH}_{2} \mathrm{CO}\right), 2.54\left(\mathrm{~s}, 3 \mathrm{H}, \mathrm{CH}_{3}\right), \\
4.57\left(\mathrm{t}, 2 \mathrm{H}, \mathrm{J} 6.8, \mathrm{NCH}_{2} \mathrm{CH}_{2}\right), \\
8.03(\mathrm{~d}, 2 \mathrm{H}, \mathrm{Ar}-\mathrm{H}), 9.03(\mathrm{~d}, 2 \mathrm{H}, \\
\text { Ar- } \mathrm{H}), 12.04\left(\mathrm{~s}, 1 \mathrm{H}, \mathrm{CO}_{2} \mathrm{H}\right)\end{array}$ & $\begin{array}{l}20.1\left(\mathrm{CH}_{3}\right), 22.5 \\
\left(\mathrm{CH}_{2}\right), 23.6\left(\mathrm{CH}_{2}\right), \\
29.1\left(\mathrm{CH}_{2}\right), 32.1 \\
\left(\mathrm{CH}_{2}\right), 58.4 \\
\left(\mathrm{NCH}_{2}\right), 127.2 \\
(\mathrm{CH}), 142.6(\mathrm{CH}), \\
157.6(\mathrm{C}), 173.1 \\
(\mathrm{CO})\end{array}$ & $\begin{array}{l}- \\
- \\
-\end{array}$ & $\begin{array}{c}3257,3131, \\
1731,1560, \\
1449,1167, \\
1031\end{array}$ & $\begin{array}{c}\left(\mathrm{M}-\mathrm{NO}_{3}\right) \\
208.22 \\
\text { found for } \\
\mathrm{C}_{12} \mathrm{H}_{18} \mathrm{NO}_{2}^{+}\end{array}$ \\
\hline
\end{tabular}




\section{Antibacterial activity}

Eight types of human pathogens were selected in order to test the in vitro antibacterial activity of some synthesized ILs (1-5). MIC values are summarized in Table V.

Table V. Antibacterial activity of ionic liquids 1-5 against eight bacterial strains

\begin{tabular}{|c|c|c|c|c|c|c|c|c|}
\hline \multirow[b]{2}{*}{ Compd. $^{a}$} & \multicolumn{8}{|c|}{$\operatorname{MIC}\left(\mathrm{mmol} \mathrm{L}^{-1}\right)$} \\
\hline & $\begin{array}{c}\text { E. } \\
\text { coli }\end{array}$ & $\begin{array}{c}\text { K. } \\
\text { pneumoniae }\end{array}$ & $\begin{array}{c}P . \\
\text { aeruginosa }\end{array}$ & $\begin{array}{c}A . \\
\text { baumannii }\end{array}$ & $\begin{array}{c}\text { S. } \\
\text { aureus }\end{array}$ & $\begin{array}{c}\text { S. } \\
\text { pneumoniae }\end{array}$ & $\begin{array}{c}B . \\
\text { subtilis }\end{array}$ & $\begin{array}{c}B . \\
\text { cereus }\end{array}$ \\
\hline 1 & $>0.97$ & $>0.97$ & $>0.97$ & $>0.97$ & $>0.97$ & $>0.97$ & $>0.97$ & $>0.97$ \\
\hline 2 & 0.40 & $>0.81$ & $>0.81$ & $>0.81$ & $>0.81$ & $>0.81$ & 0.40 & $>0.81$ \\
\hline 3 & 0.40 & 0.40 & $>0.97$ & $>0.97$ & 0.40 & 0.40 & 0.40 & $>0.97$ \\
\hline 4 & $>0.81$ & $>0.81$ & $>0.81$ & $>0.81$ & $>0.81$ & $>0.81$ & $>0.81$ & $>0.81$ \\
\hline 5 & 0.44 & 0.44 & $>0.89$ & $>0.89$ & $>0.89$ & 0.44 & $>0.89$ & $>0.89$ \\
\hline Mezlocillin & 0.24 & 0.24 & 0.24 & 0.24 & - & - & 0.059 & 0.059 \\
\hline Amikacin & 0.054 & 0.054 & 0.054 & 0.054 & 0.054 & 0.054 & - & - \\
\hline Tetracycline & 0.036 & 0.036 & - & 0.036 & - & 0.018 & 0.009 & 0.009 \\
\hline
\end{tabular}

a Solvent/negative control: water

Among the tested ILs 1-5, 1-(4-phenoxybutyl)-4-picolinium bromide (3) displayed the best antibacterial activity against $S$. pneumoniae, E. coli, K. pneumoniae, S. aureus, S. pneumoniae and B. subtilis compared to the tested antibiotics (mezlocillin, amikacin, tetracycline), which could be attributed to its phenoxylbutyl group. Furthermore, 1-(6-ethoxy-6-oxohexyl)-3-picolinium bromide (2) and 1-(5-carboxypentyl)-4-picolinium bromide (5) exhibited moderate activity, which could be attributed to the presence of the ester or carboxylic group.

MIC levels ranged was from $0.40 \mathrm{mmol} \mathrm{L}^{-1}$ to more than $0.97 \mathrm{mmol} \mathrm{L}^{-1}$ and are much higher comparable to those observed for the standard antibiotics.

\section{CONCLUSIONS}

In this study, the synthesis of a series of new, environmentally benign picolinium-based ionic liquids was successfully carried out by applying an environmentally friendly and time saving protocol. In general, improvements in rates and yield of reactions were observed by carrying out the reactions under the influence of microwave and ultrasound irradiation. The results encourage us to continue our investigations in order to find a new series of eco-friendly ionic liquids. The minimum inhibitory concentration of some tested ILs showed moderate to low activity against eight types of human pathogens.

Acknowledgements. - The author thanks Dr Wael El-sayed for carrying out the biological test of some ionic liquids.

Supplementary data. - Supplementary data $\left({ }^{1} \mathrm{H}\right.$ NMR, ${ }^{13} \mathrm{C}$ NMR, ${ }^{11} \mathrm{~B}$ NMR, ${ }^{19} \mathrm{~F}$ NMR, ${ }^{31} \mathrm{P}$ NMR) associated with this article are available upon request. 


\section{REFERENCES}

1. J. H. Davis, Jr., Task-specific ionic liquids, Chem. Lett. 33 (2004) 1072-1079; DOI: 10.1246/cl.2004.1072.

2. J. H. Wang, D. H. Cheng, X. Y. Chen, Z. Du and Z. L. Fang, Direct extraction of double stranded DNA into ionic liquid 1-butyl-3-methylimidazolium hexafluorophosphate and its quantification, Anal. Chem. 79 (2007) 620-625; DOI: 10.1021/ac061145c.

3. F. Endres, Ionic liquids: solvents for the electrodeposition of metals and semiconductors, Chem. Phys. Chem. 3 (2002) 144-154; DOI: 10.1002/1439-7641(20020215)3:2.

4. Y. F. Lin and I. W. Sun, Electrodeposition of zinc from a Lewis acidic zinc chloride-1-ethyl-3-methylimidazolium chloride molten salt, Electrochim. Acta 44 (1999) 2771-2777; DOI: 10.1016/S00134686(99)00003-1.

5. M. A. M. Ibrahim, M. Messali, Z. Moussa, A. Y. Alzahrani, S. N. Alamry and B. Hammouti, Corrosion inhibition of carbon steel by imidazolium and pyridinium cations ionic liquids in acidic environment, Port. Electrochim. Acta 29 (2013) 375-389; DOI: 10.4152/pea.201106375.

6. M. Messali, A green microwave-assisted synthesis, characterization and comparative study of new pyridazinium-based ionic liquids derivatives towards corrosion of mild steel in acidic environment, J. Mater. Environ. Sci. 2 (2011) 174-185.

7. M. Messali and M. A. M. Asiri, A green ultrasound-assisted access to some new 1-benzyl-3-(4phenoxybutyl) imidazolium-based ionic liquids derivatives - potent agents inhibitors against corrosion of mild steel in acidic environment, J. Mater. Environ. Sci. 4 (2013) 770-785.

8. A. Zarrouk, M. Messali, M. R. Aouad, H. Zarrok, R. Salghi, B. Hammouti, A. Chetouani and S. S. Al-Deyab, Some new ionic liquids derivatives: Synthesis, characterization and comparative study towards corrosion of C-steel in acidic media, J. Chem. Pharm. Res. 4 (2012) 3427-3436.

9. A. Zarrouk, M. Messali, H. Zarrok, R. Salghi, A. Al-Sheikh Ali, B. Hammouti, S. S. Al-Deyab and F. Bentiss, Synthesis, characterization and comparative study of new functionalized imidazoliumbased ionic liquids derivatives towards corrosion of C38 steel in molar hydrochloric acid, Int. J. Electrochem. Sci. 7 (2012) 6998-7015.

10. S. Takahashi, N. Koura, S. Kohara, M. L. Saboungi and L. A. Curtiss, Technological and scientific issues of room-temperature molten salts, Plasmas Ions 2 (2009) 91-105; DOI: 10.1016/S12883255(99)00105-7.

11. J. F. Brennecke and E. J. Magin, Ionic liquids: innovative fluids for chemical processing, AIChE J. 47 (2001) 2384-2389; DOI: 10.1002/aic.690471102.

12. F. Shi, Y. Gu, Q. Zhang and Y. Deng, Development of ionic liquids as green reaction media and catalysts, Catal. Surv. Asia 8 (2004) 179-186; DOI: 10.1023/B:CATS.0000038536.55980.

13. R. F. De Souza, J. C. Padilha, R. S. Goncalves and J. L. Rault-Berthelot, Dialkyl imidazolium ionic liquids as electrolytes for hydrogen production from water electrolysis, Electrochem. Commun. 8 (2006) 211-216; DOI: 10.1016/j.elecom.2005.10.036.

14. P. Kubisa, Application of ionic liquids as solvents for polymerization processes, Prog. Polym. Sci. 29 (2004) 3-12; DOI: 10.1016/j.progpolymsci.2003.10.002.

15. R. Kawano, H. Matsui, C. Matsuyama, A. Sato, M. A. B. H. Susan, N. Tanabe and M. Watanabe, High performance dye-sensitized solar cells using ionic liquids as their electrolytes, J. Photochem. Photobiol. A 164 (2004) 87-92; DOI: 10.1016/j.jphotochem.2003.12.019.

16. G. Zbancioc, A. M. Zbancioc and I. I. Mangalagiu, Ultrasound and microwave assisted synthesis of dihydroxyacetophenone derivatives with or without 1,2-diazine skeleton, Ultrason. Sonochem. 21 (2014) 802-811; DOI: 10.1016/j.ultsonch.2013.09.012.

17. D. Garella, S. Tagliapietra, V. P. Mehta, E. Van Der Eycken and G. Cravotto, Straightforward functionalization of 3,5-dichloro-2-pyrazinones under simultaneous microwave and ultrasound irradiation, Synthesis 2010, 136-140; DOI: 10.1055/s-0029-1217065. 
18. V. Bejan, D. Mantu and I. I. Mangalagiu, Ultrasound and microwave assisted synthesis of isoindolo-1,2-diazine: A comparative study, Ultrason. Sonochem. 19 (2012) 999-1002; DOI: 10.1016/j.ultsonch.2012.02.012.

19. I. I. Mangalagiu, Recent achievements in the chemistry of 1,2-diazines, Curr. Org. Chem. 15 (2011) 730-752; DOI: 10.2174/138527211794519050.

20. G. Zbancioc and I. I. Mangalagiu, Pyrrolopyridazine derivatives as blue organic luminophores: synthesis and properties. Part 2, Tetrahedron 66 (2010) 278-282; DOI: 10.1016/j.tet.2009.10.110.

21. A. Loupy, A. Petit, J. Hamelin, F. Texier-Boulier, P. Jacquault and D. Mathe, New solvent free organic synthesis using focused microwaves, Synthesis 1998, 1213-1234; DOI: 10.1055/s-1998-6083.

22. M. Messali, An efficient and green sonochemical synthesis of some new eco-friendly functionalized ionic liquids, Arab. J. Chem. 7 (2014) 63-70; DOI: 10.1016/j.arabjc.2013.08.023.

23. M. Messali and S. A. Ahmed, A green microwave-assisted synthesis of new pyridazinium-based ionic liquids as an environmentally friendly alternative, Green Sustain. Chem. 1 (2011) 70-75; DOI: $10.4236 / g s c .2011 .13012$.

24. M. Messali, A facile and green microwave-assisted synthesis of new functionalized picoliniumbased ionic liquids (in press); DOI: 10.1016/j.arabjc.2011.06.030.

25. J. R. Carreon, K. M. Stewart, K. P. Mahon, S. Shin and S. O. Kelley, Cyanine dye conjugates as probes for live cell imaging, Bioorg. Med. Chem. Lett. 17 (2007) 5182-5185; DOI: 10.1016/j. bmcl.2007.06.097.

26. M. Messali, Z. Moussa, A. Y. Alzahrani, M. Y. El-Naggar, A. S. El Douhaibi, Z. M. A. Judeh and B. Hammouti, Synthesis, characterization, antimicrobial activity of new green-chemistry-friendly ionic liquids, Chemosphere 91 (2013) 1627-1634; DOI: 10.1016/j.chemosphere.2012.12.062.

27. M. Messali, M. R. Aouad, A. A. Ali, N. Rezki, T. Ben Hadda and B. Hammouti, Synthesis, characterization, and POM analyses of novel bioactive imidazolium-based ionic liquids, Med. Chem. Res. 24 (2015) 1387-1395; DOI: 10.1007/s00044-014-1211-x.

28. Clinical and Laboratory Standards Institute (CLSI), Document M26- $A$, Methods of determining bactericidal activity of antimicrobial agents for dilution antimicrobial susceptibility tests for bacteria that grow aerobically. Approved guideline, Wayne (PA) 1999.

29. H. Nomura, Y. Isshiki, K. Sakuda, K. Sakuma and S. Kondo, The antibacterial activity of compounds isolated from Oakmoss against Legionella pneumophila and other Legionella spp, Biol. Pharm. Bull. 35 (2012) 1560-1567; DOI: 10.1007/s11064-012-0751-z.

30. Clinical and Laboratory Standards Institute (CLSI), Document M7-A5, Methods for antibacterial susceptibility test for bacteria that grow aerobically. Approved standard, 5th ed., Wayne (PA) 2000. 